\title{
Zonas de relevo de falla en el margen oriental de la fosa del Jiloca (Cordillera Ibérica): geometría, cinemática y modelización analógica
}

\author{
Alba Peiro, José Luis Simón y Teresa Román-Berdiel \\ Dpto. de Ciencias de la Tierra, Grupo Geotransfer - Instituto de Investigación en Ciencias Ambientales (IUCA), \\ Universidad de Zaragoza, Pedro Cerbuna 12, 50009 Zaragoza, Spain \\ apeiro@unizar.es, jsimon@unizar.es,mtdjrb@unizar.es
}

\begin{abstract}
RESUMEN
El margen oriental de la fosa del Jiloca (Cordillera Ibérica) es el resultado de la disposición escalonada diestra de tres fallas normales de dirección NW-SE: Ias fallas de Calamocha, Sierra Palomera y Concud. Junto con la falla deTeruel, delimitan tres zonas de relevo con evidencias de fracturación reciente. El análisis de la deformación a escala cartográfica y de afloramiento, y su comparación con la fracturación desarrollada en varios modelos analógicos, ha permitido inferir su relación geométrica, cinemática y dinámica con las macroestructuras, en el marco del campo de esfuerzos de extensión radial o 'multidireccional' activo en la región de estudio. En los modelos analógicos se han estudiado las variaciones en el patrón de fracturación resultante, en función de las velocidades de extensión en dos direcciones ortogonales y de la orientación de las fallas principales respecto a dichas direcciones de extensión. Tanto en el área de estudio como en los modelos, la fracturación es longitudinal a las macroestructuras que delimitan los relevos. Está controlada por la dirección de las fallas $y$, en mayor medida, por la dirección de extensión ENE-WSW dominante en el campo de esfuerzos regional. Todo ello permite definir un nuevo tipo de interacción en zonas de relevo de falla extensionales, denominada en este trabajo relevo con fracturación longitudinal distribuida.
\end{abstract}

Palabras clave: campo de esfuerzos, Cuaternario, extensión radial, falla, Neógeno.

\section{Fault relay zones within the eastern sector of the Jiloca Basin (Iberian Chain): geometry, kinematics and analogue modelling}

\begin{abstract}
The eastern sector of the Jiloca basin (Iberian Chain) developed due to the right-relay arrangement of three NW-SE striking normal faults: the Calamocha, Sierra Palomera and Concud faults. Together with the Teruel fault, they all bound three relay zones where evidence of recent fracturing has been found. Thanks to the analysis of brittle deformation, at both map and outcrop scales, and to its comparison with results from analogue modelling, we are able to infer its geometrical, kinematical and dynamical relationship with the macrostructures, within the framework of the near-multidirectional extension regime active in the studied area. During analogue modelling it was important to analyse possible variations in the resulting fractures, controlled by different extension velocities in two orthogonal directions and by the orientation of the main faults within both directions of extension. The fractures, both in the studied area and in analogue models, show a mostly parallel orientation to the macrostructures that bound the relay zones. They are controlled by the main fault strikes and, to a greater extent, by the dominant ENE-WSW direction of dominant extension within the regional stress field. All this has allowed us to define a new kind of interaction within extensional fault relay zones, called in this study relay with along-strike distributed fractures.
\end{abstract}

Keywords: fault, Neogene, Quaternary, radial extension, stress field. 
Alba Peiro, et al., 2019. Zonas de relevo de falla en el margen oriental de la fosa del... Boletín Geológico y Minero, 130 (3): $393-415$

\section{Introducción}

Las fosas neógeno-cuaternarias de la Cordillera Ibérica centro-oriental representan la deformación intraplaca asociada al rifting del Surco de Valencia. La del Jiloca es una de las fosas principales, y su relleno está afectado por tres fallas normales de dirección NW-SE: las fallas de Concud, Sierra Palomera y Calamocha (Fig. 1). Estas fallas, junto con la deTeruel, delimitan tres zonas de relevo diestro con evidencias de fracturación reciente.

El campo extensional activo en la región de estudio es de tipo radial o 'multidireccional', donde el eje $\sigma_{1}$ es vertical y $\sigma_{2}$ y $\sigma_{3}$ tienen un valor similar en la horizontal (Simón, 1989). Sin embargo, se identifica una dirección dominante del eje primario de extensión $\sigma_{3}$ próxima a ENE-WSW (Herraiz et al., 2000; Arlegui et al., 2005; Ezquerro, 2017; Liesa et al., 2019), así como numerosos casos de desviaciones e intercambios de ejes.

Resulta difícil identificar cuál ha sido la influencia real de las fallas heredadas y cuál la del campo de esfuerzos en la evolución estructural de las zonas de relevo. Es por ello que el objetivo principal de este trabajo es aportar información sobre los procesos de fracturación que se producen en las zonas de relevo de fallas normales, y profundizar, mediante el análisis estructural y la modelización analógica, en las relaciones geométricas, cinemáticas y dinámicas entre esta fracturación a diversas escalas y las fallas mayores que determinan los relevos. Para ello, este trabajo aborda el estudio desde dos aspectos: (1) el análisis estructural de las zonas de relevo entre las principales fallas del margen oriental de la fosa del Jiloca y su articulación con la de Teruel; (2) la realización de una serie de modelos analógicos que simulen la deformación producida bajo una extensión similar a la que ha operado en este sector de la Cordillera Ibérica durante el periodo tectónico reciente, a fin de identificar y analizar las variables que controlan el desarrollo de la fracturación en las zonas de relevo entre fallas y su entorno.

Los antecedentes en modelización analógica de zonas de relevo de falla comprenden únicamente experimentos desarrollados bajo extensión 'uniaxial' (e.g., Clifton et al., 2000; Mansfield y Cartwright, 2001; Le Calvez et al., 2002; Acocella et al., 2005; Hus et al., 2005). Este trabajo es probablemente el primero en el que se realizan modelos analógicos bajo una extensión 'biaxial' en dos direcciones ortogonales, lo cual implica simular una mayor variedad de condiciones estructurales y dinámicas.

\section{Contexto geológico}

\section{La fosa del Jiloca: fallas principales que delimitan su margen oriental y su articulación con la fosa de Teruel}

La fosa del Jiloca constituye una depresión alargada de unos $70 \mathrm{~km}$ de longitud y entre 6 y $12 \mathrm{~km}$ de anchu$\mathrm{ra}$, y presenta una dirección NNW-SSE, cortando la directriz NW-SE de las estructuras contractivas de la cadena (Fig. 1). El hundimiento y el consiguiente relleno de la fosa del Jiloca comenzaron a finales del Plioceno, durante el periodo extensional vinculado al rifting del Surco de Valencia. Su estructura resultó claramente asimétrica: su límite occidental presenta un suave basculamiento hacia el este y se articula mediante un sistema de fallas escalonado, mientras que su margen oriental es el resultado directo de la disposición escalonada diestra de tres grandes fallas normales NW-SE. Se trata de las fallas de Calamocha, Sierra Palomera y Concud (Simón, 1983, 1989).

La falla de Teruel, es una falla normal de dirección $\mathrm{N}-\mathrm{S}$ a NNE-SSW que se extiende unos $9 \mathrm{~km}$ en el interior de la Cuenca de Teruel. Su salto neto es de aproximadamente $270 \mathrm{~m}$ y su tasa de desplazamiento de 0,075 mm/año (Simón et al., 2017). Las estrías de deslizamiento indican una dirección media de transporte hacia N $275^{\circ} \mathrm{E}$ (Simón et al., 2017). Su bloque hundido también presenta una estructura en roll-over, más marcado que en el caso de la falla de Concud (Lafuente et al., 2011).

La falla de Concud se dispone en dirección NW-SE, aunque en su sector más meridional se orienta más NNW-SSE, aproximándose a la dirección de la falla de Teruel. Su longitud es de $14,2 \mathrm{~km}$ y las estrías de deslizamiento indican una dirección de transporte hacia $\mathrm{N} 220^{\circ} \mathrm{E}$. Con un salto neto de $255-300 \mathrm{~m}$, presenta un extenso pliegue de roll-over en su bloque hundido al que, en las inmediaciones de la falla, se le añade un sinclinal de acomodación (Lafuente et al., 2011). Se trata de una falla activa que ha mantenido una tasa media de movimiento de 0,07-0,08 mm/año desde el Plioceno medio (Lafuente et al., 2011) y de 0,29 $\mathrm{mm} / \mathrm{año}$ durante el Pleistoceno Superior (Simón et al., 2016).

La falla de Sierra Palomera es una falla normal de $15,6 \mathrm{~km}$ de longitud, con dirección general NNW-SSE, asociada al flanco oriental de un pliegue anticlinal vergente al E. En su plano de falla se han identificado estrías de deslizamiento que indican una dirección de transporte hacia N $255^{\circ} \mathrm{E}$ (García-Lacosta et al., 2014). Los estudios más recientes muestran cómo muchos de sus rasgos morfológicos sugieren un grado de actividad reciente notable (García-Lacosta et al., 2014), y permiten estimar un salto neto desde el 


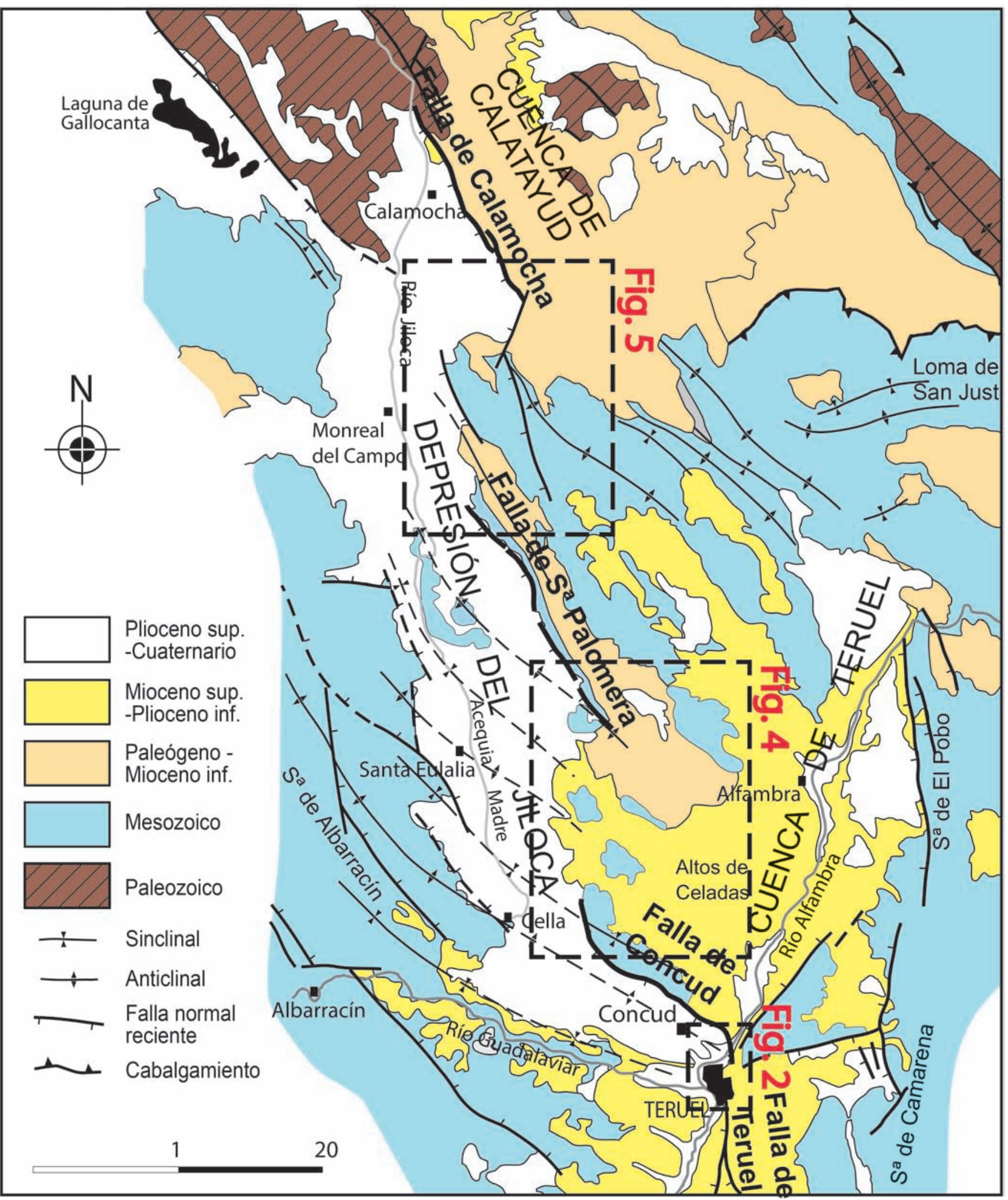

Figura 1. Fosas de Teruel y del Jiloca y su situación dentro de la Península Ibérica. Se señalan las zonas de relevo entre las fallas deTeruel, Concud, Sierra Palomera y Calamocha (Fig. 2, 4 y 5, respectivamente).

Figure 1. The Teruel and Jiloca basins and their location within the Iberian Peninsula. The relay zones between the Teruel, Concud, Sierra Palomera and Calamocha Faults are marked (Figs. 2, 4 and 5, respectively). 
Plioceno medio de unos 350-400 m (Rubio y Simón, 2007).

La falla de Calamocha alcanza una longitud de 17 $\mathrm{km}$, también con una dirección general NNW-SSE. En su sector central las estrías de deslizamiento presentan cabeceos de aproximadamente $90^{\circ}$. En este mismo sector se ha calculado un desplazamiento neto máximo de $220 \mathrm{~m}$ (Martín-Bello et al., 2014). Su tasa de movimiento se ha mantenido constante desde el Plioceno superior entre 0,06 y 0,09 mm/año y presenta claras evidencias de actividad cuaternaria (Simón, 1983; Simón et al., 2012). Su sector central se divide en varias superficies de rotura sintéticas, entre las que también aparecen fallas antitéticas N-S, cuya distribución y orientación sugiere la presencia de una estructura en roll-over en su bloque superior.

\section{Estratigrafía}

Las unidades mesozoicas de la Cadena Ibérica han sido moldeadas por diferentes etapas orogénicas: desde la formación y posterior inversión negativa del Rift Ibérico o Aulacógeno Ibérico durante la Orogenia Alpina (Álvaro et al., 1979) hasta la extensión neógeno-cuaternaria que daría lugar a las actuales fosas del Jiloca y Teruel (Simón, 1982). Es por ello que dichos materiales presentan un alto grado de deformación y están parcialmente cubiertos, en los bloques levantados, y cubiertos casi en su totalidad por materiales neógenos y cuaternarios en los fondos de las fosas del Jiloca y Teruel.

El encajamiento fluvial en la fosa del Jiloca es casi nulo, lo cual dificulta conocer las características de su relleno. A partir de varios sondeos, Rubio y Simón (2007) constatan la existencia de espesores de relleno sedimentario de la fosa que llegan a superar los 100 $\mathrm{m}$. Estos autores distinguen tres grandes unidades sobre el sustrato mesozoico: Unidad 1 o inferior (Mioceno superior-Plioceno superior; de carácter carbonatado), Unidad 2 o intermedia (Plioceno superior; detrítica, que culmina en superficie con el sistema de glacis villafranquienses) y Unidad 3 o superior (Cuaternario; de carácter detrítico y origen aluvial, que en ocasiones arranca desde los escarpes de falla).

La estratigrafía de la zona de articulación con la fosa Teruel es más conocida porque aflora debido a la incisión de los valles del Guadalaviar y del Turia. Se compone de varias unidades informales (Godoy et al., 1983): Rojo 1 (Vallesiense; arcillas rojas con intercalaciones de conglomerados y areniscas), Páramo 1 (Turoliense; carbonatos lacustres blanquecinos), Rojo 2 (Turoliense-Rusciniense; arcillas rojo-anaranjadas), Páramo 2 (Rusciniense; carbonatos lacustres blan- quecinos) y Rojo 3 (Rusciniense-Villafranquiense; arcillas rojas). Esta última queda cubierta hacia el oeste por el sistema de glacis villafranquienses. Los materiales cuaternarios se depositaron formando cuatro niveles de terrazas fluviales, además de un conjunto de abanicos aluviales que, al igual que en la fosa del Jiloca, parten desde los escarpes de falla más importantes.

\section{Análisis estructural de la fracturación La zona de relevo entre las fallas de Concud y Teruel}

La zona de relevo entre las fallas de Concud y Teruel es un área de aproximadamente $10 \mathrm{~km}^{2}$. Su disposición en relevo diestro obliga a que el bloque intermedio entre ambas acomode la transferencia de desplazamiento mediante un basculamiento hacia el $\mathrm{N}$ o NNW (relay ramp), visible en la zona de El Planizar (ver mapa de la Fig. 2). Se trata de un área afectada por un conjunto de fallas, orientadas NNW-SSE $y$, ocasionalmente, NNE-SSW, alineadas con la posible prolongación hacia el norte de la traza principal de la falla de Teruel (Fig. 2; Peiro, 2016; Peiro et al., 2017).

En el sector norte de la zona de relevo (afloramiento 1 en Fig. 2) la Terraza Media (Pleistoceno Medio) y a la unidad Rojo 3 (Plioceno Superior; Fig. $3 \mathrm{~A}, \mathrm{~B})$ están afectadas por el siguiente conjunto de estructuras: una zona de falla NNE-SSW (estereograma 1 b en Fig. 2), un conjunto de fallas menores NNWSSE y numerosas fracturas (estereogramas $1 a$, c en Fig. 2). Todas las fallas integradas producen un salto aparente total de unos $18 \mathrm{~m}$ en la base de la terraza (Peiro, 2016). Las estrías medidas en dos planos de falla indican una dirección de transporte común del bloque superior hacia ENE (estereogramas $1 a$, b en Fig. 2).

Más al sur (afloramiento 2 en Fig. 2) se observan tres fallas afectando tanto a la Terraza Media como a los materiales villafranquienses subyacentes, todas ellas de dirección NNW-SSE. Dos de estas fallas producen saltos aparentes métricos y decamétricos en la terraza pleistocena, basculando los bloques intermedios entre ellas. La más importante de ambas presenta una orientación 157, $48 \mathrm{~W}$ y estrías con cabeceo 70 $S$ (Simón et al., 2017). En la parte oriental aflora un tercer plano de rotura con un salto aparente de $3 \mathrm{~m}$.

Por último, el afloramiento 3 (Fig. 2) muestra en su sector noreste la Terraza Inferior (Pleistoceno Superior) afectada por una zona de falla principal de dirección $138,53 \mathrm{~W}$ y salto aparente mínimo de 2,4 m, así como por un conjunto de fallas y fracturas menores sintéticas y antitéticas (estereograma 3 en Fig. 2). Todo ello se interpreta como un sistema de fallas nor- 
males conjugadas a partir del cual, aplicando el modelo de Anderson (1951), se infiere un sistema de esfuerzos hipotético con un $\sigma_{1}$ prácticamente vertical y $\sigma_{3}$ orientado SW-NE, si bien al carecer de datos de estrías esta interpretación no es del todo robusta. En su sector suroeste se ha inferido la presencia de otra falla que desplaza la Terraza Media un mínimo de unos $7 \mathrm{~m}$, con una estructura en roll-over asociada a su bloque hundido.

\section{La zona de relevo entre las fallas de Sierra Palomera y Concud}

El área comprendida entre las terminaciones sur y norte de las fallas de Sierra Palomera y Concud, respectivamente, alcanza una extensión de aproximadamente $200 \mathrm{~km}^{2}$. En su sector norte (afloramiento 1 en Fig. 4) afloran las arcillas rojas y el glacis villafranquiense correspondientes a la Unidad 2, afectados

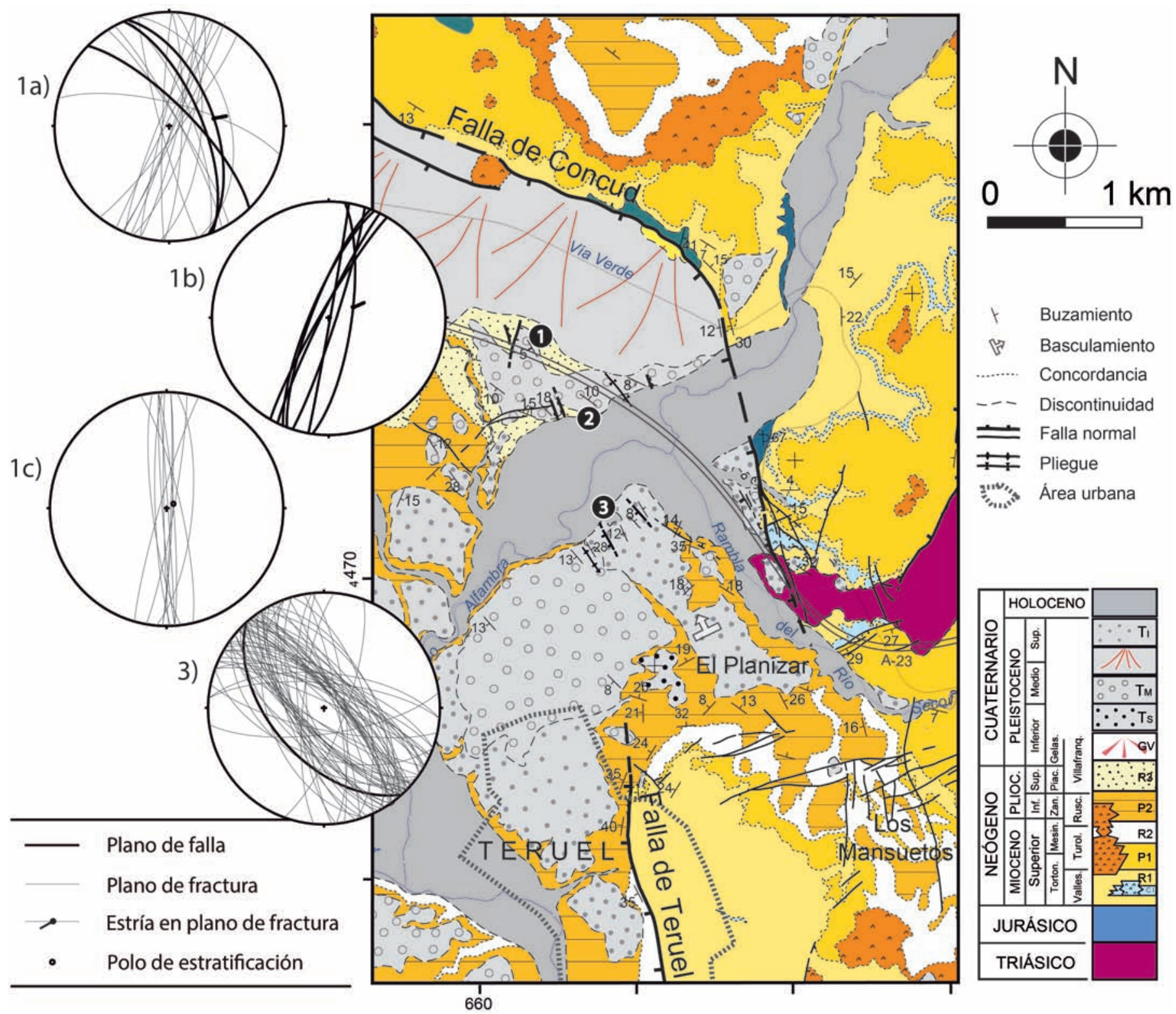

Figura 2. Zona de relevo entre las fallas de Concud y Teruel (modificado de Lafuente, 2011). 1a-3) Estereogramas de direcciones de fracturas medidas en los diferentes afloramientos.

Figure 2. Relay zone between the Concud and Teruel Faults (modified from Lafuente, 2011). 1a-3) Stereograms of fracture directions measured at different sites. 

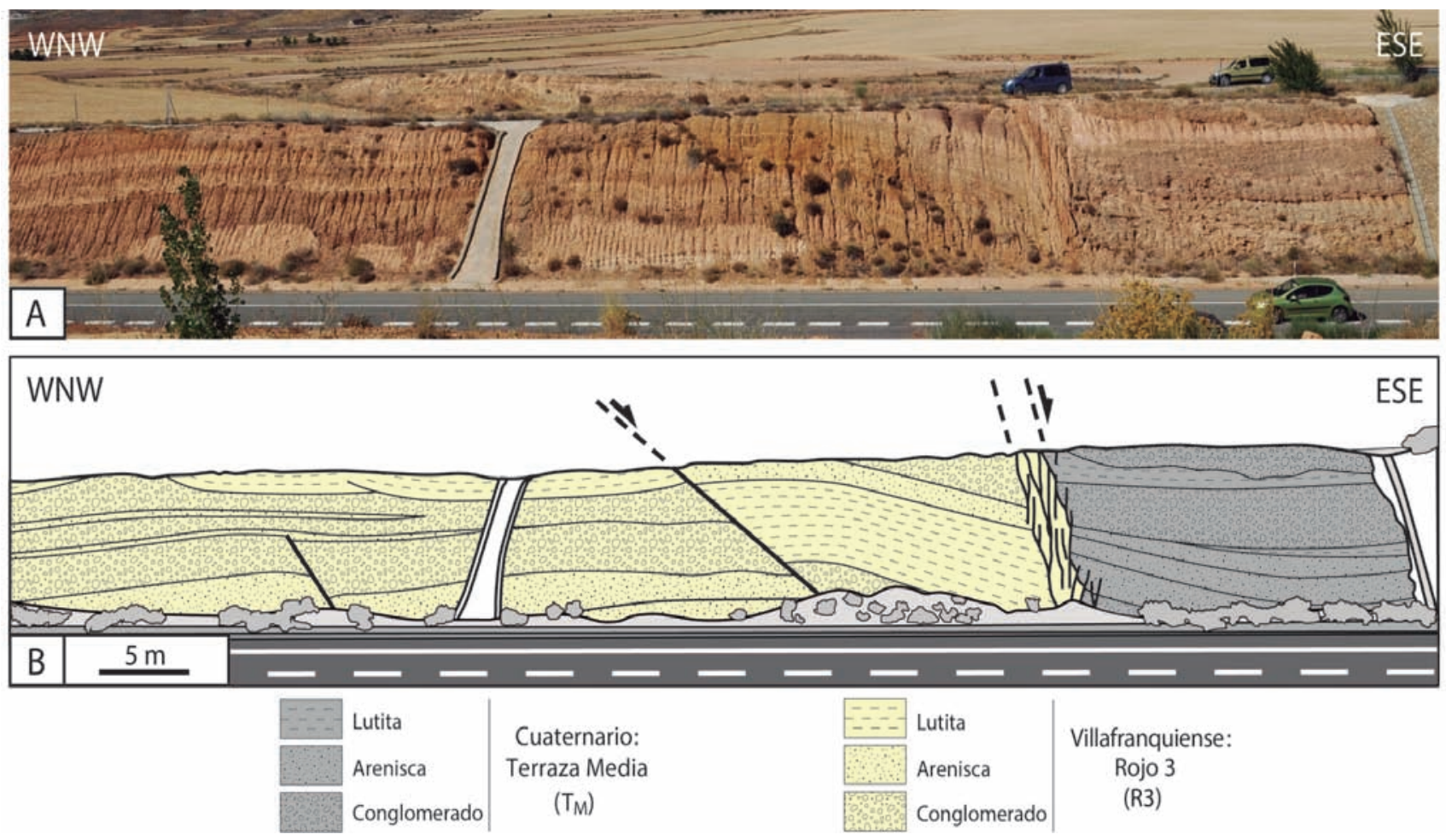

Figure 3. A) y B) Fallas normales afectando a la unidad Rojo 3 y a la Terraza Media en el afloramiento 1 (punto 1 en la Fig. 2). Figure 3. A) and B) Normal faults cutting the Rojo 3 unit and the Middle Terrace at site 1 (number 1 in Fig. 2).

por numerosas fracturas menores. Éstas se disponen con una orientación preferente NNW-SSE, en las arciIlas, y N-S, en el Villafranquiense (estereogramas 1 a y b en Fig. 4, respectivamente; Simón, 1983). En este mismo punto el Villafranquiense también está afectado por fallas N-S, con saltos aparentes decimétricos y métricos. Más al sur se aprecia otra falla vertical con dirección $\mathrm{N} 144^{\circ} \mathrm{E}$ que también corta a la Unidad 2 (afloramiento 2 y estereograma 2 en Fig. 4). Su salto no es visible en el afloramiento.

El afloramiento 3 (Fig. 4) se compone de varios paquetes conglomeráticos correspondientes al Mioceno Superior de la Unidad 1 afectados por un conjunto de fracturas, mayoritariamente verticales $y$ orientadas en dirección N-S (estereograma 3 en Fig. 4).

Finalmente, los afloramientos 4 y 5 (Fig. 4) están visiblemente fracturados a escala cartográfica (Ezquerro, 2017). Los diagramas en rosa de las fallas y fracturas correspondientes muestran una clara dominancia de la dirección N-S (diagrama en rosa 4 en Fig. 4), y NW-SE y su perpendicular (diagrama en rosa 5 en Fig. 4), aunque de forma menos acusada en este último caso.

\section{La zona de relevo entre las fallas de Calamocha y Sierra Palomera}

La última zona de relevo es la situada más al norte, que alcanza una extensión de más de $150 \mathrm{~km}^{2}$. En su sector central se encuentra un conjunto de pequeñas fosas delimitadas por fallas de dirección N-S a NNWSSE (Fig. 5). Sólo existe un afloramiento claro de uno de esos planos de falla, el mostrado en la Fig. 6A, cuyo descubrimiento en los años 80 permitió la interpretación tectónica de este conjunto de depresiones, que Gutiérrez et al. (1983) describen como valles tectónicos. En otros puntos se observan pequeños escarpes de entre 1 y $2 \mathrm{~m}$ de altura sobre el fondo del valle que se atribuyen a planos de falla degradados. También hay constancia de estructuras de interacción entre estos escalones de falla, como son las rampas de relevo.

En el sector oeste de la zona de relevo (afloramiento 1 en Fig. 5), los conglomerados del Mioceno Superior correspondientes a la Unidad 1 están fracturados a escala entre centimétrica y decamétrica con desplazamientos visibles en campo (Fig. 6B, C). En la cartografía y en el diagrama en rosa (Fig. 6B y dia- 


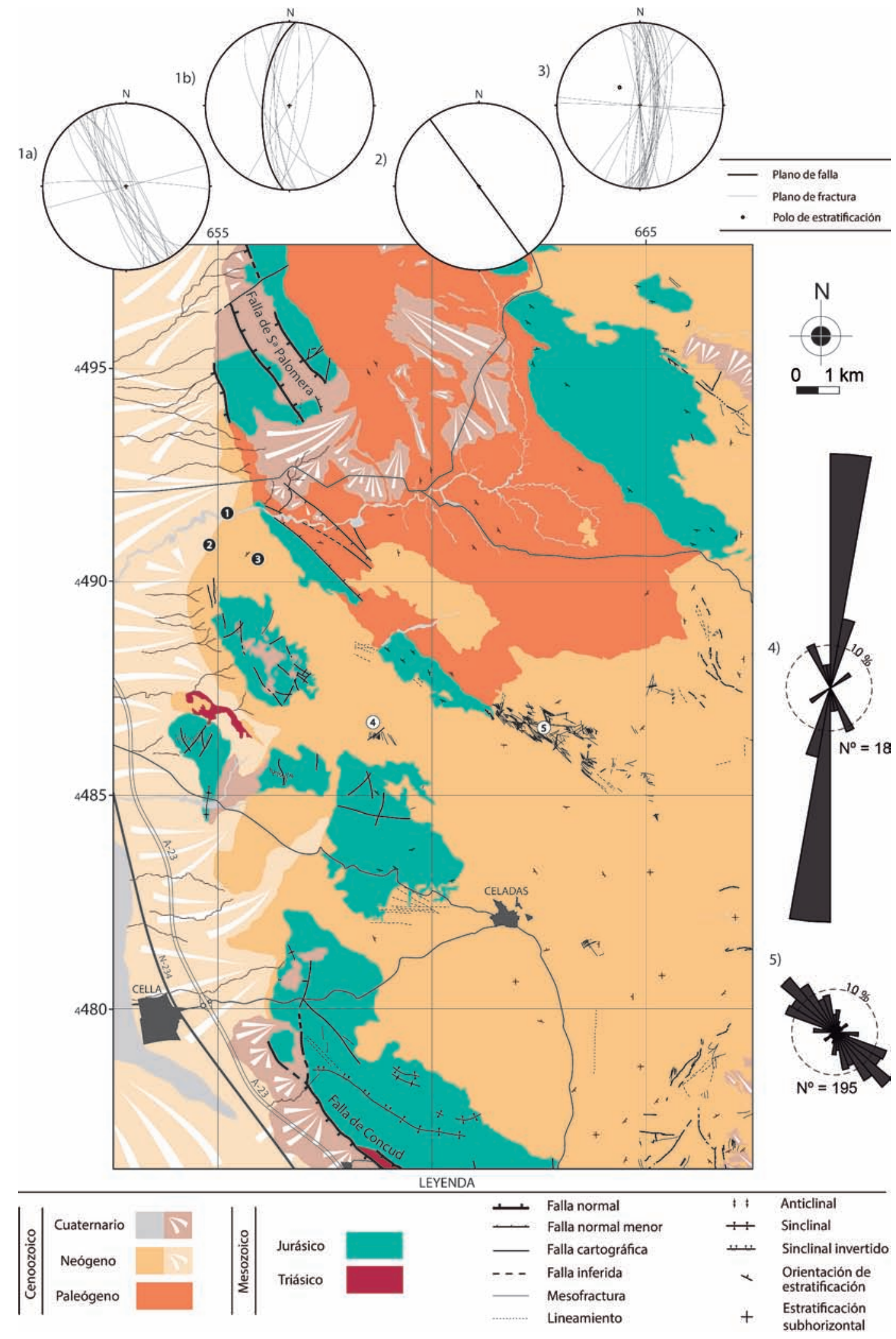

Figura 4. Zona de relevo entre las fallas de Sierra Palomera y Concud. 1a-5) Estereogramas y diagramas en rosa con las direcciones de fracturas medidas en los distintos afloramientos. La cartografía de las fallas de los afloramientos 4 y 5 ha sido tomada de Ezquerro (2017). Figure 4. Relay zone between the Sierra Palomera and Concud Faults. 1a-5) Stereograms and rose diagrams with fracture directions measured at different sites. Fault mapping of sites 4 and 5 has been taken from Ezquerro (2017). 


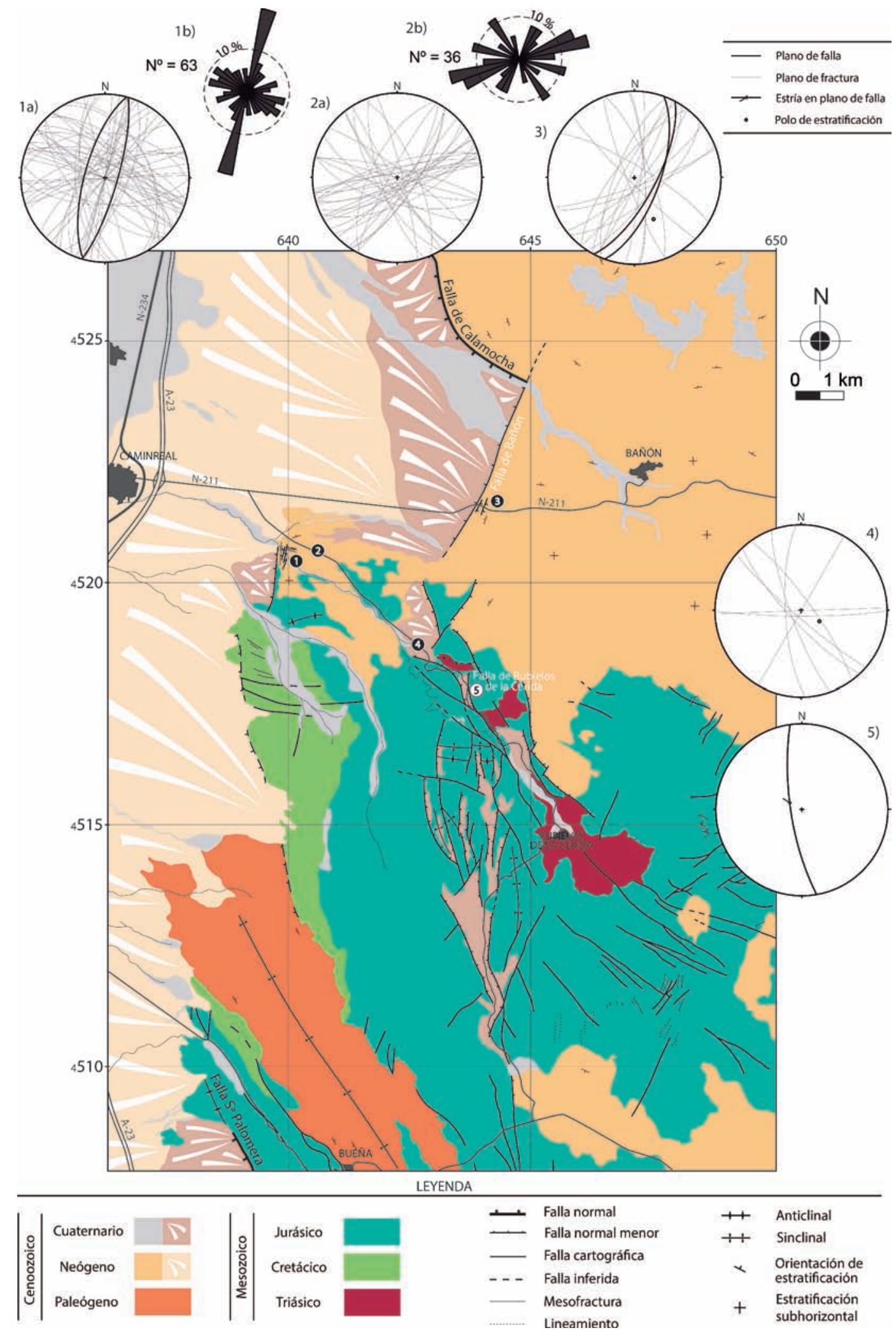

Figura 5. Zona de relevo entre las fallas de Calamocha y Sierra Palomera. 1a-5) Estereogramas y diagrama en rosa con las direcciones de fracturas medidas en los distintos afloramientos. El afloramiento 5 ya fue estudiado por Gutiérrez et al. (1983).

Figure 5. Relay zone between the Calamocha and Sierra Palomera Faults. 1a-5) Stereograms and rose diagram with fracture directions measured at different sites. Site 5 was also studied by Gutiérrez et al. (1983). 
grama 1b en Fig. 5) de la fracturación se diferencian dos familias: una en $\mathrm{N} 015^{\circ} \mathrm{E}$ y su perpendicular, más dispersa, en torno a $\mathrm{N} 100^{\circ} \mathrm{E}$. Otro tramo de esta misma unidad aparece fuertemente fracturado en el afloramiento 2 (Fig. 5). Se trata de fracturas de escala decimétrica que se disponen en varias direcciones preferentes (estereograma 2a y diagrama en rosa $2 b$ en Fig. 5): una mayoritaria ENE-WSW y su ortogonal NNW-SSE, y otras dos también ortogonales entre sí, NNE-SSW y ESE-WNW.

Respecto a la falla de Bañón (afloramiento 3 en Fig. 5), situada en la parte norte, la información que se tiene a día de hoy se reduce a aquella inferida de la cartografía MAGNA (Hernández et al., 1983; Olivé et al., 1983) como es su dirección $\mathrm{N} 025^{\circ} \mathrm{E}$. En campo su plano no aflora, pero sí algunas de las estructuras asociadas a ella: dos fallas antitéticas a la principal, un par sintéticas relacionadas con las anteriores, así como numerosas fracturas (estereograma 3 de la Fig. 5).

El afloramiento 4 (Fig. 5) es un afloramiento con evidencias de fracturación en los materiales aluviales cuaternarios de la Unidad 3 (afloramiento y estereograma 4 en Fig. 5). Las orientaciones son dispersas, aunque con una ligera predominancia de la dirección NNW-SSE a NW-SE.

La falla de Rubielos de la Cérida aparece expuesta en un afloramiento excepcional (Capote et al., 1981; Gutiérrez et al., 2008) y pone en contacto materiales jurásicos y cuaternarios (afloramiento 5 en Fig. 5; Fig. 6A). Estos últimos están datados mediante $\mathrm{C} 14$ entre $>48,5$ ka y 43,1 ka (Gutiérrez et al., 2008). Presenta estrías y otros marcadores cinemáticos (estereogra- ma 5 en Fig. 5). Su dirección es cercana a N-S, al igual que los grábenes en los que se encuentra, y la media de las estrías de deslizamiento indica una dirección de transporte hacia $\mathrm{N} 300^{\circ} \mathrm{E}$.

\section{Modelos analógicos bajo régimen de extensión ‘bia- xial' $^{\prime}$}

\section{Dispositivo experimental y materiales utilizados}

El dispositivo experimental se compone de una lámina cuadrangular de látex que reposa sobre la mesa de modelización y constituye la base del modelo (Fig. 7). La lámina de látex es similar a la utilizada en otros trabajos para investigar el efecto de un estiramiento uniforme, aplicado en la base del modelo (i.e. sin discontinuidad de velocidad), sobre el desarrollo de las fallas (e. g., Vendeville et al., 1987; Hus et al., 2005). Dicha lámina está sujeta a dos pistones de madera móviles dispuestos ortogonales, y está fija en los otros dos extremos. El movimiento de estos pistones se lleva a cabo mediante dos sistemas de motores (M1 y M2). Sobre la lámina de látex se dispone el modelo constituido por una capa de arena que cubre por completo una banda de silicona. La configuración escalonada en planta (Tabla 1) de esta banda de silicona es la que permite la formación de zonas de relevo, y se basa en la empleada por Hus et al. (2005). La deformación puede visualizarse y cuantificarse gracias una cuadrícula trazada sobre la superficie del modelo con un espaciado de $5 \times 5 \mathrm{~cm}$.

Los modelos presentados en este trabajo se reali-
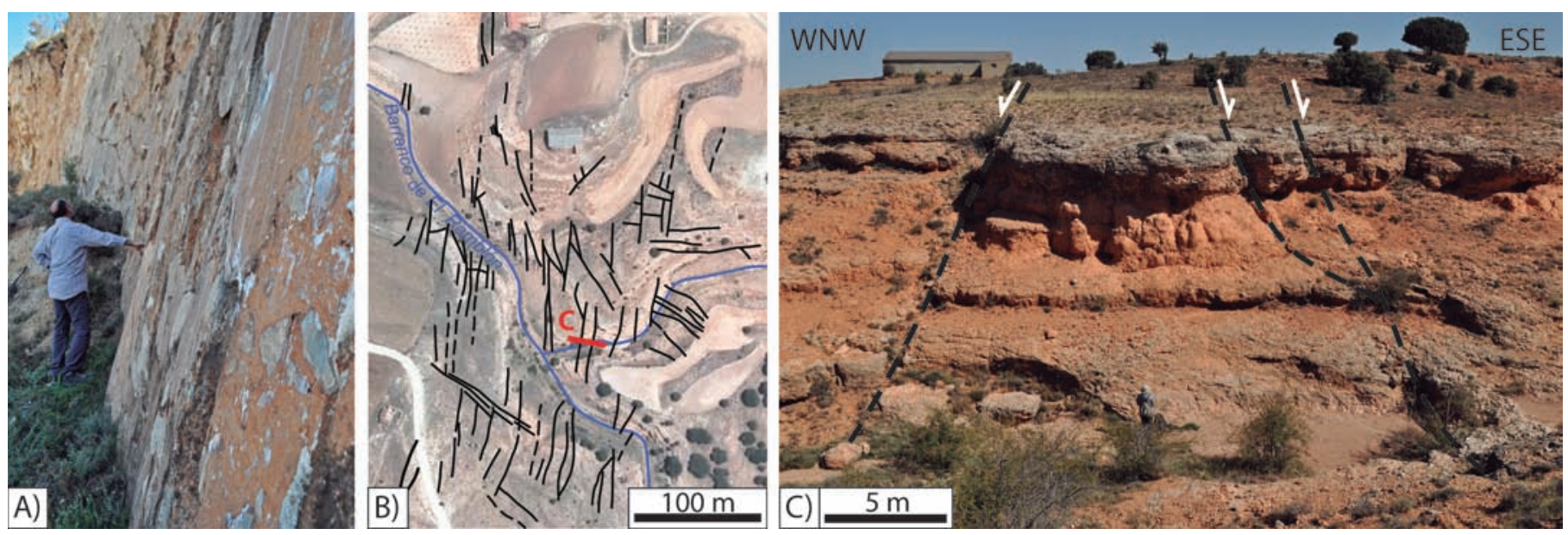

Figura 6. A) Plano expuesto de la falla de Rubielos de la Cérida (punto 5 en Fig. 5). B) Mapa de detalle de la fracturación en el afloramiento 1 de la Fig. 5. C) Fallas normales en la Unidad 1, se señala su localización en B.

Figure 6. A) The Rubielos de la Cérida Fault plane (site 5 in Fig. 5). B) Detailed fracture map from site 1 in Fig. 5. C) Normal faults cutting the Unit 1, its location is marked in $B$. 


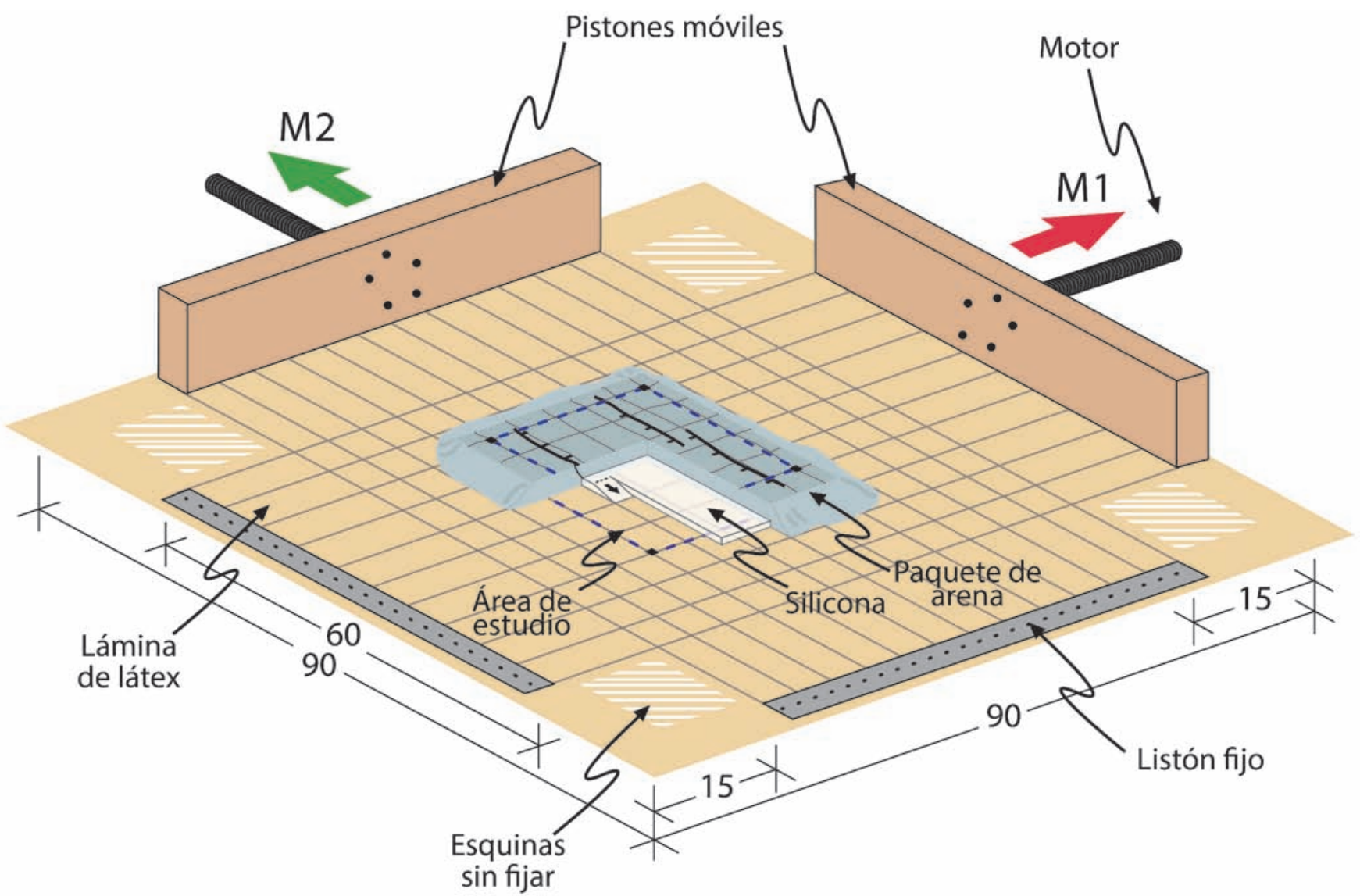

Figura 7. Dispositivo experimental. Las longitudes señaladas están expresadas en centímetros.

Figure 7. Experimental set-up. Lengths are expressed in centimetres.

zan en campo gravitatorio normal y son representativos de la corteza superior, o de parte de ella, en un área sometida a extensión y sin respuesta isostática. Para representar el comportamiento frágil de los materiales de la corteza superior se utilizan habitualmente materiales granulares que se deforman de acuerdo con el comportamiento de tipo MohrCoulomb (Mandl, 1977; Krantz, 1991; Schellart, 2000). En concreto, para representar el comportamiento frágil de los materiales mesozoicos y cenozoicos del área de estudio se ha utilizado Arena Silícea L-70/80 S de Sibelco Hispania, con una composición de 99\% de $\mathrm{SiO}_{2}$, granulometría entre 100 y $500 \mu$, densidad de $1500 \mathrm{~kg} \mathrm{~m}^{-3}$, ángulo de rozamiento interno de $32^{\circ} \mathrm{y}$ cohesión nula. Aunque la finalidad principal de la banda de silicona es que sus bordes sirvan para nuclear las fallas principales y permitan la formación de zonas de relevo (Hus et al., 2005), también puede ser representativa de los niveles dúctiles del Triásico medio-superior, nivel que funciona como nivel de despegue en la Cordillera Ibérica y que aflora en algu- nos puntos de la zona estudiada. Es común utilizar siliconas para representar el comportamiento de este tipo de niveles de baja resistencia (Weijermars y Schmeling, 1986; Vendeville et al., 1987). En nuestro caso, para representar los materiales del Triásico Superior, se ha utilizado silicona incolora de tipo Rhodorsil Gomme FB de Caldic, que presenta una viscosidad de $7 \times 10^{3} \mathrm{~Pa}$ s a $20^{\circ} \mathrm{C}$ y una densidad de $980 \mathrm{~kg}$ $\mathrm{m}^{-3}$.

\section{Tipos de modelos realizados}

Se han realizado un total de diez experimentos en condiciones de extensión 'biaxial' (Tabla 1): cuatro de ellos (R1, R2, R3 y R4) son modelos de referencia para estudiar el comportamiento de los materiales por separado (en R1 y R2 se estudia el campo de deformación que se genera en la lámina de látex y que se aplicará en las series 1 y 2 , respectivamente); la Serie 1 (B1-1, B1-2, B1-3) y la Serie 2 (B2-1, B2-2, B2-3). Para 
Alba Peiro, et al., 2019. Zonas de relevo de falla en el margen oriental de la fosa del... Boletín Geológico y Minero, 130 (3): $393-415$

\begin{tabular}{|c|c|c|c|c|c|c|c|c|c|c|c|c|}
\hline \multirow{3}{*}{\multicolumn{2}{|c|}{ MODELO }} & \multicolumn{6}{|c|}{ DISEÑO } & \multicolumn{5}{|c|}{ EXTENSIÓN } \\
\hline & & \multicolumn{3}{|c|}{ Espesores } & \multicolumn{2}{|c|}{ Silicona } & \multirow{2}{*}{ 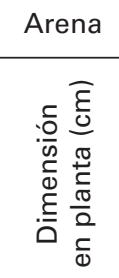 } & \multicolumn{3}{|c|}{ Velocidad } & \multicolumn{2}{|c|}{ Motores } \\
\hline & & \multirow{2}{*}{$\begin{array}{l}\overline{\frac{\varepsilon}{0}} \\
\bar{\sigma} \\
\overline{0} \\
.0 \\
\overline{\bar{\omega}} \\
-\end{array}$} & \multirow{2}{*}{ 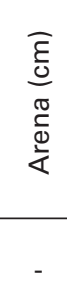 } & \multirow{2}{*}{ 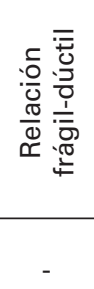 } & \multirow{2}{*}{$\begin{array}{l}\frac{0}{0} \\
\frac{0}{0} \\
\frac{0}{0} \\
\Sigma \\
\end{array}$} & \multirow{2}{*}{ 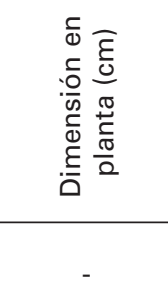 } & & \multirow{2}{*}{ 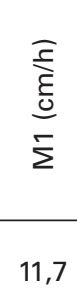 } & \multirow{2}{*}{ 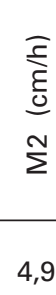 } & \multirow{2}{*}{$\begin{array}{l}\underset{\sum}{\stackrel{o}{\varrho}} \\
\stackrel{\sum}{\Sigma}\end{array}$} & \multirow{2}{*}{$\begin{array}{l}\frac{\overline{\underline{\varepsilon}}}{\bar{\Sigma}} \\
20\end{array}$} & \multirow{2}{*}{$\begin{array}{l}\frac{\overline{\underline{E}}}{\bar{N}} \\
9\end{array}$} \\
\hline Serie 1 & R1 & & & & & & - & & & & & \\
\hline Serie 2 & R2 & - & - & - & - & - & - & 10,1 & 8 & $\sim 80$ & 14 & 12,5 \\
\hline \multicolumn{2}{|c|}{ R3 } & - & 2 & - & - & - & $50 \times 50$ & 11,7 & 5,8 & 50 & 15,3 & 7,5 \\
\hline \multicolumn{2}{|c|}{ R4 } & 0,5 & 1,5 & 3 & & $50 \times 50$ & $50 \times 50$ & 11,7 & 5,8 & 50 & 15,3 & 7,5 \\
\hline \multirow{3}{*}{ Serie 1} & B1-1 & 0,5 & 1 & 2 & & $\sim 25 \times 10,8$ & $25 \times 25$ & 11,7 & 4,9 & $\sim 40$ & 20 & 9 \\
\hline & B1-2 & 0,5 & 1 & 2 & & $\sim 25 \times 10,8$ & $25 \times 25$ & 11,7 & 4,9 & $\sim 40$ & 20 & 9 \\
\hline & B1-3 & 0,5 & 1 & 2 & & $\sim 25 \times 10,8$ & $25 \times 25$ & 11,7 & 4,9 & $\sim 40$ & 20 & 9 \\
\hline \multirow{3}{*}{ Serie 2} & B2-1 & 0,5 & 1 & 2 & & $\sim 25 \times 10,8$ & $25 \times 25$ & 10,1 & 8 & $\sim 80$ & 14 & 12,5 \\
\hline & B2-2 & 0,5 & 1 & 2 & & $\sim 25 \times 10,8$ & $25 \times 25$ & 10,1 & 8 & $\sim 80$ & 14 & 12,5 \\
\hline & B2-3 & 0,5 & 1 & 2 & & $\sim 25 \times 10,8$ & $25 \times 25$ & 10,1 & 8 & $\sim 80$ & 14 & 12,5 \\
\hline
\end{tabular}

Tabla 1. Tabla resumen de las características de los modelos analógicos.

Table 1. Summarizing table of the analogue models. characteristics.

cada serie se aplica una relación diferente entre las dos velocidades de extensión ortogonales $y$, dentro de cada serie, los modelos se diferencian en la orientación de la banda basal de silicona (Tabla 1). Los bordes de la silicona actúan como "fallas maestras", heredadas, con orientación variable respecto a las trayectorias de extensión, de manera que pueda analizarse la influencia de esa orientación en el desarrollo y evolución de las zonas de relevo.

\section{Dimensionamiento}

Simular cualquier proceso natural mediante la modelización analógica implica dimensionar previamente los modelos respecto al prototipo en la naturaleza siguiendo los principios de Hubbert (1937) y Ramberg (1981) de similitud geométrica, cinemática y dinámica. Para ello, se ha elegido una relación de longitudes $L=1,25 \times 10^{-5}$, lo que significa que $1 \mathrm{~cm}$ en los modelos equivale a $0,8 \mathrm{~km}$ en la naturaleza (Tabla 2). El campo gravitatorio, densidades y viscosidades vienen impuestas por las condiciones experimentales y por los materiales utilizados. Por lo tanto, el factor de escala del campo gravitatorio es $\mathrm{g}=1$, ya que los modelos se realizan en campo gravitatorio normal, y la relación de densidades entre la naturaleza (considerando los rangos de valores de densidades para calizas compactas y para yesos; Sharma, 1997) y los materiales empleados es $\rho \sim 0,5$ (Tabla 2). La proporcionalidad entre las fuerzas tectónicas y gravitacionales es $\sigma=\rho \mathrm{g} L \sim 6,25 \times 10^{-6} \mathrm{y}$ el factor de escala para 
Alba Peiro, et al., 2019. Zonas de relevo de falla en el margen oriental de la fosa del... Boletín Geológico y Minero, 130 (3): $393-415$

\begin{tabular}{|c|c|c|c|}
\hline PARÁMETRO & Modelo & Naturaleza & Relación Modelo/Naturaleza \\
\hline Longitud $(\mathrm{cm})$ & 1 & $8 \times 10^{4}$ & $1,25 \times 10^{-5}$ \\
\hline Densidad de la capa frágil $\left(\mathrm{kg} \mathrm{m}^{-3}\right)$ & 1500 & 2700 & $\sim 0,5$ \\
\hline Densidad de la capa dúctil $\left(\mathrm{kg} \mathrm{m}^{-3}\right)$ & 980 & $1 \times 10^{19}$ (Koyi, 1988) & $\sim 0,5$ \\
\hline Viscosidad de la capa dúctil (Pa s) & $7 \times 10^{3}$ & $31^{\circ}-40^{\circ}$ (Bahroudi et al., 2003) & $7 \times 10^{-16}$ \\
\hline Ángulo de rozamiento interno & $32^{\circ}$ & $8,89 \times 10^{9}$ & $\sim 1$ \\
\hline Tiempo (h) & 1 & $7,2 \times 10^{-5}$ & $1,12 \times 10^{-10}$ \\
\hline Velocidad $\left(\mathrm{cm} \mathrm{h}^{-1}\right)$ & 8 & $1,11 \times 10^{5}$ \\
\hline
\end{tabular}

Tabla 2. Parámetros del dimensionamiento entre los modelos y la naturaleza.

Table 2. Scaling parameters for models and nature.

la viscosidad del nivel dúctil $\mu=7 \times 10^{-16}$ (considerando una viscosidad de $10^{19} \mathrm{~Pa}$ s para el nivel de baja resistencia en la naturaleza; Koyi, 1988; Tabla 2). La relación temporal es $\mathrm{T}=1,12 \times 10^{-10}(1 \mathrm{~h}$ de experimento representa $1 \mathrm{Ma}$ en la naturaleza) y la relación de velocidades $V=1,11 \times 10^{5}(1 \mathrm{~cm} / \mathrm{h}$ en el laboratorio representan 0,79 $\mathrm{mm} /$ año en la naturaleza). La arena es un material que responde a la Ley de MohrCoulomb $(\tau=\sigma \operatorname{tg} \varphi+C)$, con ángulos de rozamiento interno $(\varphi)$ muy similares a los de la naturaleza y cohesión (C) nula. La silicona presenta un comportamiento dúctil de tipo newtoniano $(\sigma=\mu \varepsilon)$ que permite simular la plasticidad de ese posible nivel de despegue arcilloso-salino (Weijermars y Schmeling, 1986).

\section{Comportamiento general de los modelos: resultados preliminares}

El análisis de los cuatro modelos de referencia (R1, R2, R3 y R4) ha permitido estudiar el comportamiento de los materiales por separado. En el modelo formado sólo por arena (R3) no se formaron estructuras. Bajo las mismas condiciones, el modelo R4 dio como resultado un complejo sistema de fracturación compuesto mayoritariamente por grábenes, aunque también se formaron fallas aisladas y otras estructuras asociadas a los bordes de la silicona. El uso de esta capa dúctil parece ser, por tanto, un punto clave para inducir la nucleación de estructuras en la arena.

Para conocer en profundidad el campo de deformación que afecta a los modelos de las series 1 y 2 se analizó la extensión experimentada por la lámina de látex en los modelos R1 (Fig. 8A) y R2 (Fig. 8B), llevados a cabo sin arena ni silicona. Dicha extensión quedó registrada mediante marcadores circulares trazados previamente sobre la lámina de látex. Conforme avanzaban los experimentos los marcado- res circulares se transformaron dando lugar a un conjunto de elipses de deformación, a partir de las cuales se han reconstruido las trayectorias de máxima extensión. La interpretación dinámica de la fracturación resultante en cada experimento se basa en el supuesto de que el campo de deformación transmitido a la capa de arena reproduce ese campo de deformación de la lámina de látex (eje de esfuerzo mínimo $\sigma_{3}$ paralelo al eje $X$ de deformación). Ese paralelismo entre trayectorias de deformación y esfuerzo puede asumirse si la deformación finita es moderada y no existe una componente rotacional significativa.

La diferencia de extensión entre los motores M1 y M2 es el doble en el modelo R1 y, por tanto, en la Serie 1, que en el modelo R2 y en la Serie 2 (Tabla 1). La Serie 2 representa unas condiciones de esfuerzo más próximas a la extensión 'radial' y muestra, a la vez, una menor homogeneidad en las condiciones de deformación impuestas, con trayectorias más curvadas (Fig. 8B) que en la Serie 1 (Fig. 8A).

\section{Estudio sistemático de los modelos}

Para los análisis de las series 1 y 2 se ha diferenciado entre las estructuras generadas en la parte central de los modelos (donde existe silicona en su base), fundamentalmente grábenes (denominados en adelante grábenes centrales), y aquellas asociadas a los bordes de la silicona (fallas de borde), que han sido las fallas principales y otros grábenes. La nucleación de estos dos grupos de estructuras tienen un origen diferente: en el primer caso es el movimiento de la silicona bajo el campo extensional y la ascensión de diapiros en su interfaz con la arena, mientras que en el segundo es el propio borde de la silicona.

Gracias a la toma de fotografías a intervalos de tiempo regulares en los experimentos de las Series 1 y 2 , se ha podido medir tanto el ángulo respecto al 
A) R1
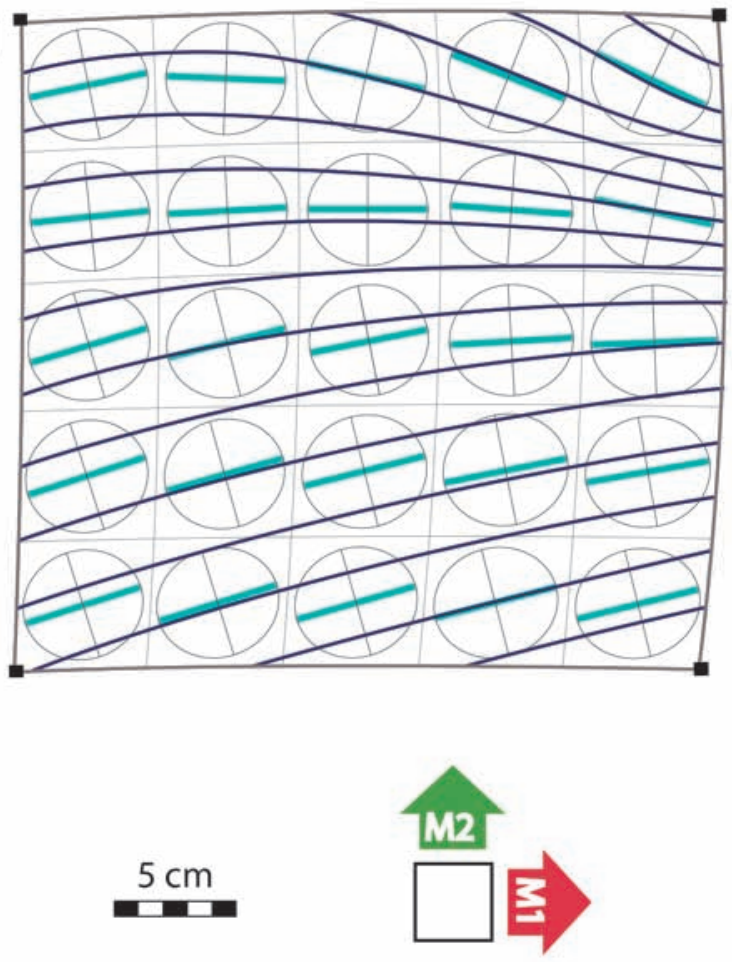

B) $\mathrm{R} 2$

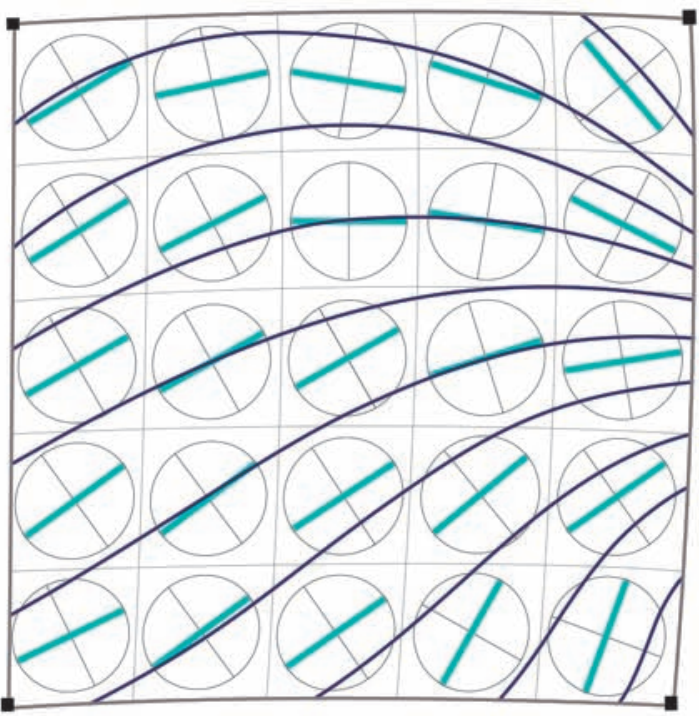

Cuadrícula y límite del sector de estudio

Elipses de deformación

Eje X (extensión máxima)

Trayectoria de la extensión máxima

Figura 8. Análisis de los modelos R1 (A) y R2 (B). Se señala la posición de los motores M1 y M2.

Figure 8. Analysis of models R1 (A) and R2 (B). The position of motors M1 and M2 is also marked.

norte $(\alpha)$ como el ángulo respecto a la dirección de extensión $\sigma_{3}(\beta)$. Estas mediciones sistemáticas han permitido calcular dos ángulos de desviación (Fig. 9): el ángulo $\theta$ entre la dirección de los bordes de la silicona y de las estructuras, y el ángulo $\gamma$ entre la dirección de $\sigma_{3}$ y la perpendicular a las estructuras. Para conocer la influencia relativa que han tenido el borde de la silicona y el campo de deformación impuesto, se considerarán estructuras controladas por los bordes de la silicona aquellas cuyo ángulo $\theta$ sea menor que $\pm 20^{\circ}$, y controladas con el campo de deformación aquellas cuyo ángulo $\gamma$ sea menor que $\pm 20^{\circ}$.

\section{Serie 1: B1-1, B1-2, B1-3}

Los modelos de la Serie 1 (Fig. 10A, B y C) se caracterizan por un desarrollo abundante de fracturación, sobre todo de grábenes centrales. En el caso de las fallas de borde, éstas se manifiestan claramente en B1-1 y B1-2 (Fig. 10A, B, respectivamente), pero con menor desarrollo en B1-3 (Fig. 10C). La oblicuidad del borde de la silicona respecto al campo de deformación influye, en gran medida, en la generación de este tipo de fracturas.

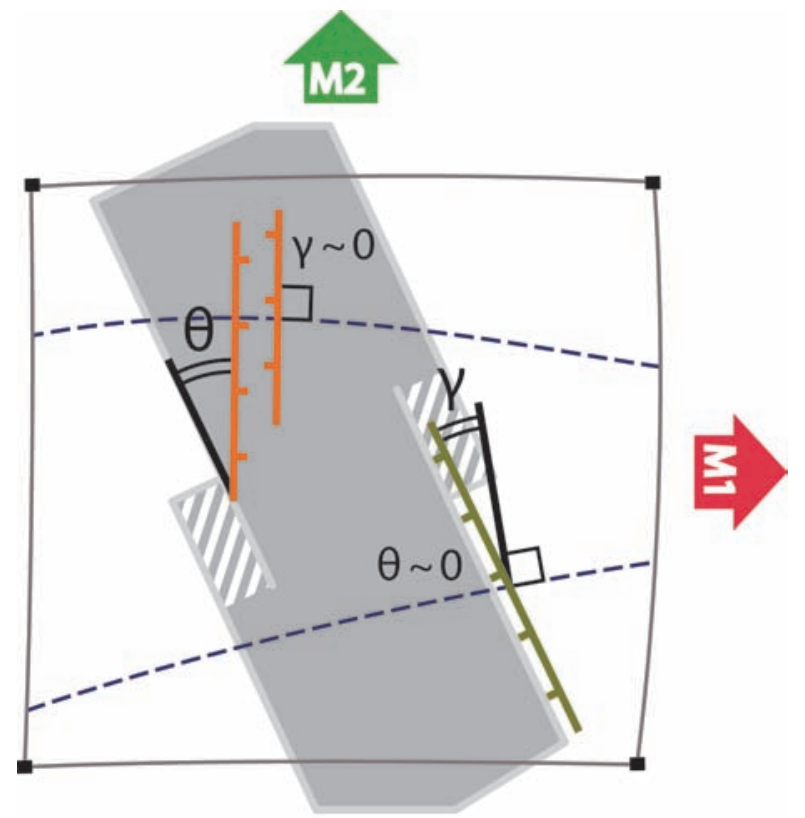

Figura 9. Ángulos aplicados en el análisis de las estructuras tipo graben ( $\theta$; parte superior), y en el de aquellas nucleadas sobre las "fallas maestras" ( $\gamma$; parte inferior).

Figure 9. Angles used at the analysis of graben-like structures ( $\theta$; upper part), and of those nucleated over the "master faults" ( $\gamma$; lower part). 

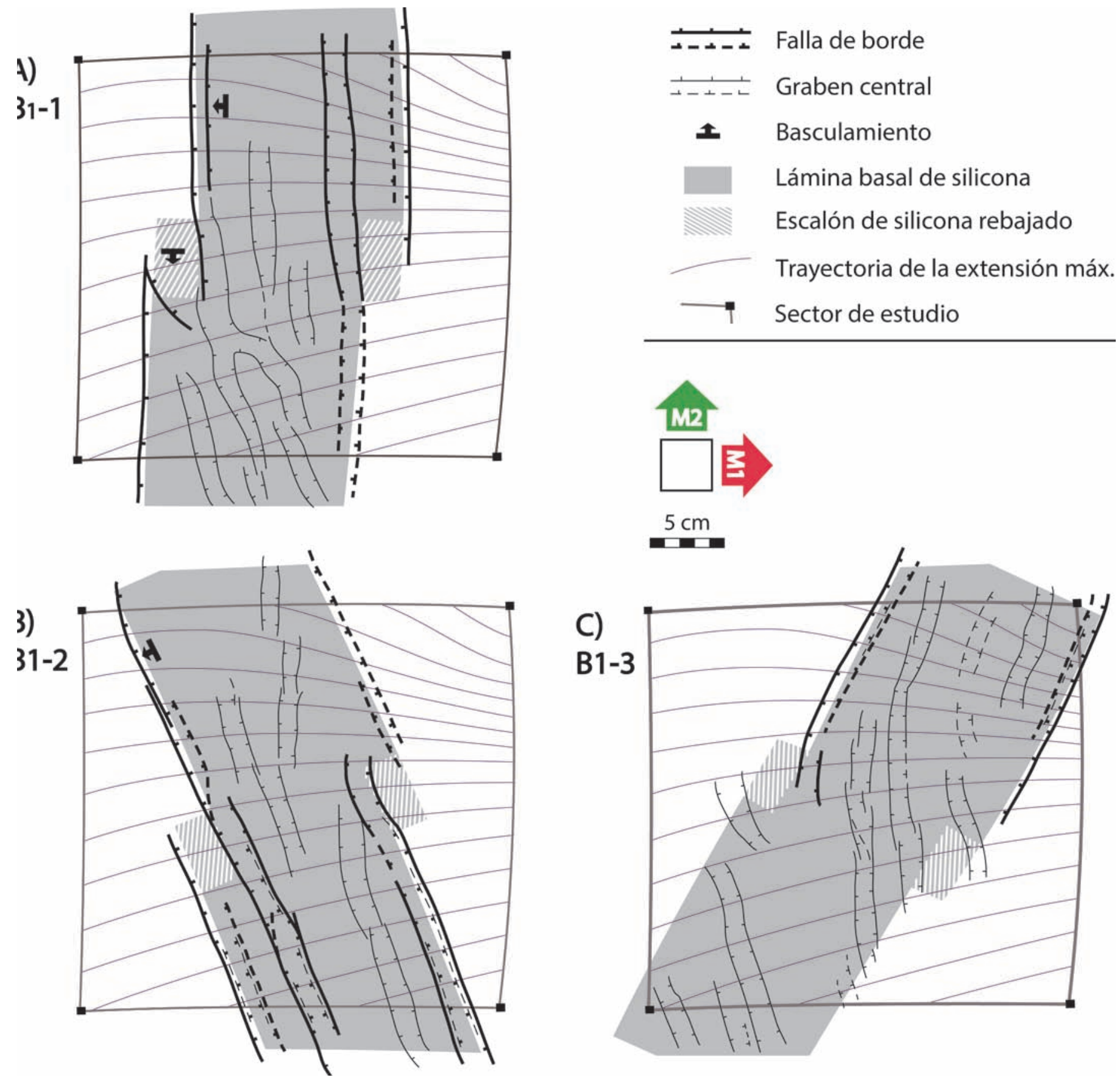

Figura 10. Análisis final de los modelos de la Serie 1: B1-1 (A), B1-2 (B) y B1-3 (C). Figure 10. Final analysis of models from Series 1: B1-1 (A), B1-2 (B) y B1-3 (C).

La abundancia de estructuras formadas en la Serie 1 ha permitido analizar estadísticamente cuál ha sido el control del borde de la silicona y del campo de deformación impuesto en la formación y crecimiento de los grábenes centrales y de las fallas de borde (Fig. 11). Es lógico comprobar cómo, en todos los casos, la compatibilidad con la dirección del campo de defor- mación es mayor en los grábenes centrales, y con el borde de la silicona en las fallas de borde. Cuando la morfología de la silicona es aproximadamente ortogonal a la dirección de extensión (modelo B1-1; Fig. $10 \mathrm{~A}, 11 \mathrm{~A})$, los grábenes centrales presentan también compatibilidad con los bordes de la silicona. Cuando es oblicua (modelos B1-2 y B1-3; Fig. 10B, C; Fig. 11B, 


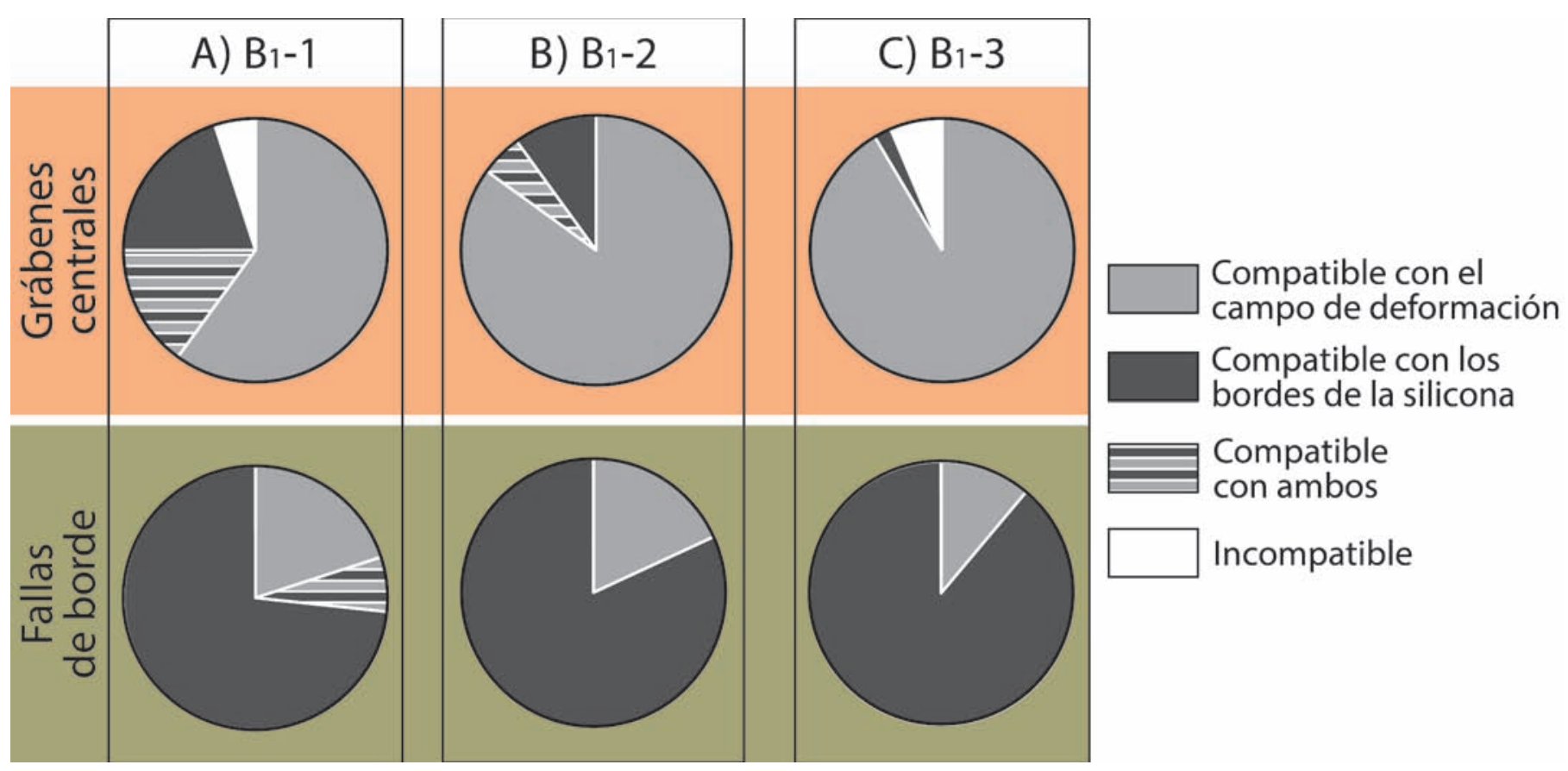

Figura 11. Compatibilidad de las estructuras con el campo de deformación y el borde de la silicona en los modelos B1-1 (A), B1-2 (B) y B13 (C), calculada a partir de los ángulos $\theta$ y $\gamma$.

Figure 11. Compatibility of structures with the deformation field and the silicone boundaries in models B1-1 (A), B1-2 (B) y B1-3 (C), calculated from angles $\theta$ and $\gamma$.

C), esta compatibilidad disminuye, mientras que el número de grábenes controlados por el campo de deformación aumenta. Las fallas de borde presentan este mismo patrón: cuando los bordes de la silicona son ortogonales (modelo B1-1; Fig. 10A, 11A) las fallas de borde son también compatibles con el campo de deformación, pero cuando es oblicua (modelos B1-2 y B1-3; Fig. 10B, C; Fig. 11B, C) este control disminuye, acentuándose la influencia de la dirección de los bordes. En todas las estructuras del borde se ha comprobado que las fallas correspondientes a los porcentajes compatibles con el campo de deformación (Fig. 11) siempre se desarrollan dentro de las zonas de relevo.

\section{Serie 2: B2-1, B2-2 y B2-3}

En los modelos de la Serie 2 (Fig. 12A, B y C) la presencia de grábenes centrales es prácticamente nula; en consecuencia, no se han realizado los análisis de compatibilidad descritos anteriormente. Por el contrario, las fallas de borde sí que están bien desarrolladas en los tres modelos. De nuevo vuelve a manifestarse mejor en los casos B2-1 y B2-2 (Fig. 12A y B) que en B2-3 (Fig. 12C).

En la Fig. 13 se observa cómo, además de la evidente compatibilidad con la dirección del borde de la silicona, también hay un porcentaje alto de estructuras de borde compatibles con el campo de deformación en los tres modelos. Estos valores corresponden a las estructuras de interacción que se desarrollan entre las fallas principales, y que se comentan en el siguiente apartado.

\section{Interacción entre fracturas}

En el caso de los grábenes centrales, se ha constatado la existencia de varios modos de interacción: i) bifurcaciones de los planos de falla dentro de un mismo graben (ejemplos en los modelos B1-2 y B1-3), ii) uniones directas entre varios grábenes (ejemplos en R-3 y B1-1), iii) uniones por rampa de relevo entre dos grábenes (ejemplos en R-3, B1-1 y B1-2).

Las fallas principales, desarrolladas a partir de los bordes de la lámina de silicona, han desarrollado importantes sistemas de interacción, como por ejemplo basculamientos de relay ramp (ejemplos en B1-1, B2-1, ver Fig. 14A, y en B2-2 y B2-3) o prolongaciones con geometría "en gancho". Estas últimas se forman en zonas próximas al escalón de silicona y presentan una marcada curvatura que se adapta bien al campo de deformación (ejemplos en B1-2, B1-3, B2-1, B2-2, ver Fig. 14B, y en B2-3). Asimismo, se han generado 

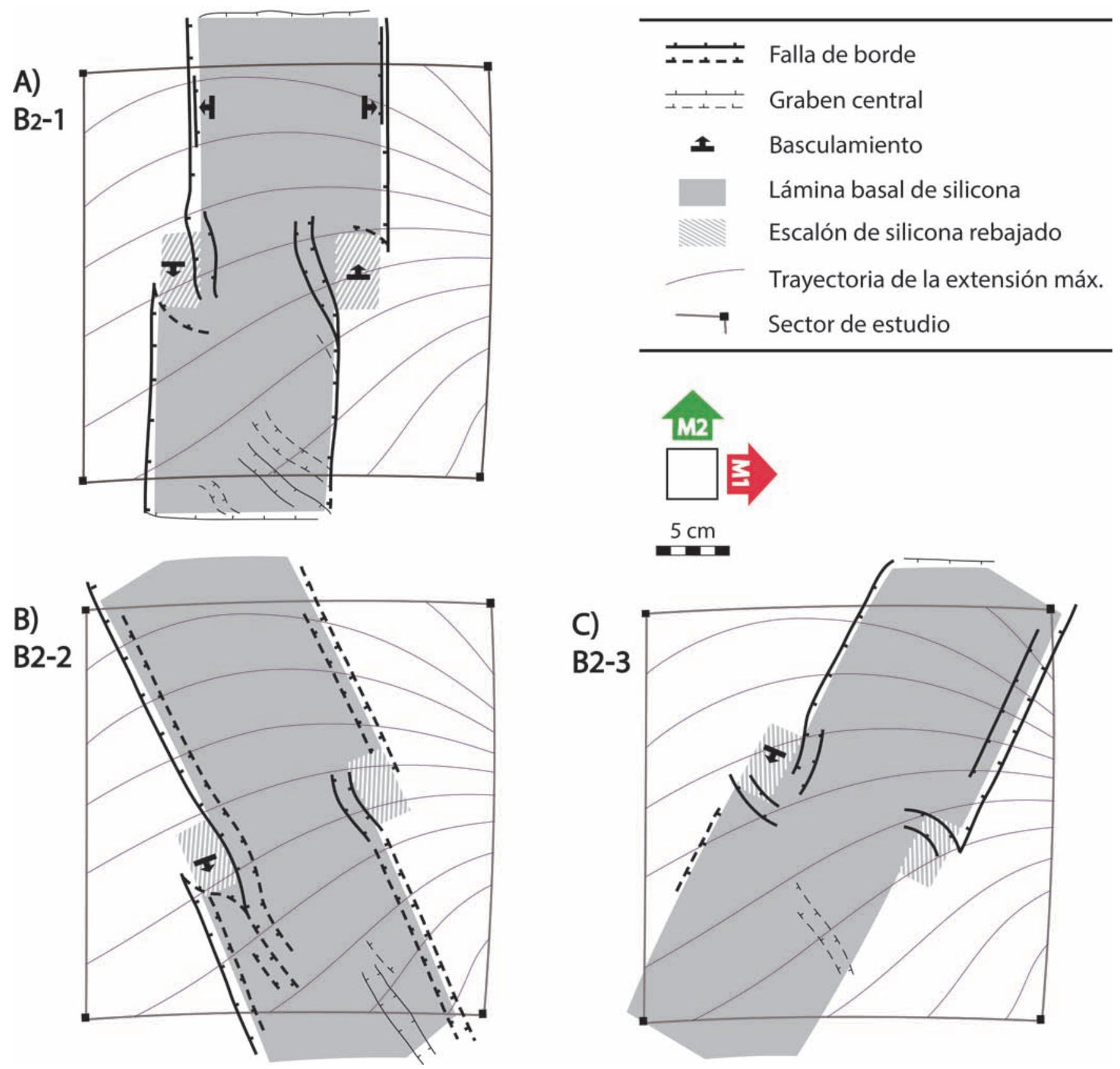

Figura 12. Análisis final de los modelos de la Serie 2: B2-1 (A), B2-2 (B) y B2-3 (C).

Figure 12. Final analysis of models from Series 2: B2-1 (A), B2-2 (B) y B2-3 (C).

estructuras transversales a la dirección de las fallas principales, formadas en estadios avanzados de los experimentos. Se ha visto que la dirección de crecimiento de estas últimas siempre está más influenciada por el campo de deformación que por la orientación de los bordes de la silicona (ejemplos en B1-1, B2-1, B2-2, ver Fig. 14C, y en B2-3).

\section{Interpretación y discusión} La fracturación en las zonas de relevo del margen
oriental de la fosa del Jiloca

La disposición en relevo diestro de las fallas deTeruel, Concud, Sierra Palomera y Calamocha hace que los 


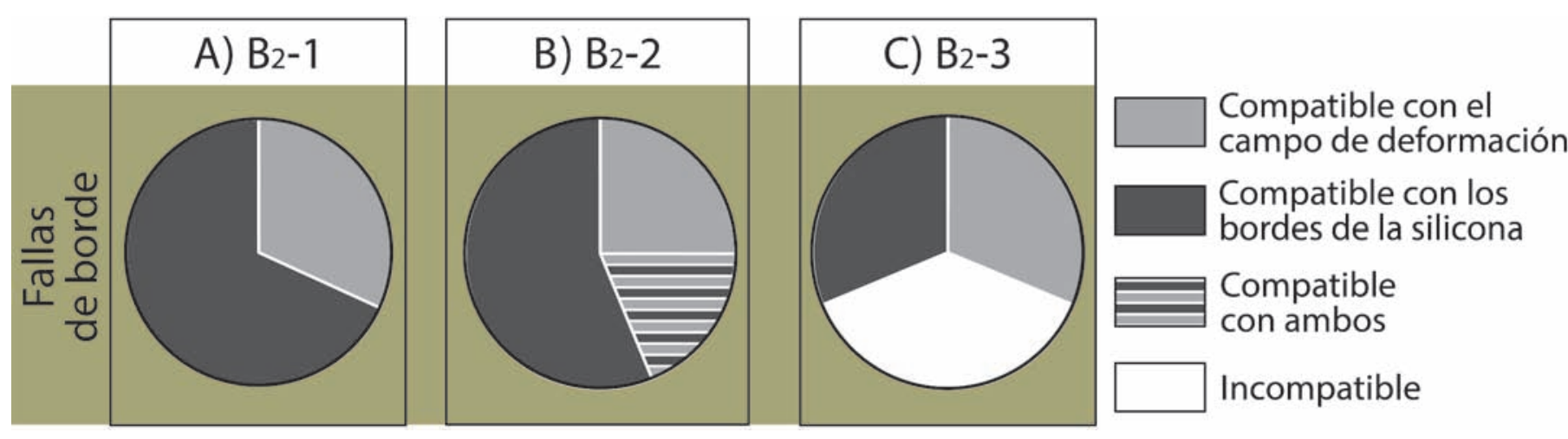

Figura 13. Compatibilidad de las estructuras con el campo de deformación y el borde de la silicona en los modelos B2-1 (A), B2-2 (B) y B23 (C), calculada a partir de los ángulos $\theta$ y $\gamma$.

Figure 13. Compatibility of structures with the deformation field and the silicone boundaries in models $B 2-1(A), B 2-2(B)$ y $B 2-3(C)$, calculated from angles $\theta$ and $\gamma$.

bloques intermedios entre ellas acomoden la transferencia de desplazamiento mediante una rampa de relevo (relay ramp), mecanismo característico del denominado soft linkage (Walsh y Watterson, 1991). En la zona de relevo entre las fallas de Concud y Teruel esa rampa de relevo es más acusada y se expresa como un basculamiento visible hacia el $\mathrm{N}$ o NNW (Fig. 2), mientras que en las otras dos zonas (mucho más amplias debido a la distancia entre los respectivos extremos de las fallas) se aprecian caídas tenues hacia el NW (Fig. 4 y 5).

Las evidencias de fracturación reciente están repartidas de manera bastante homogénea en las tres zonas de relevo. Las fallas y fracturas que cortan la zona de relevo entre las fallas de Concud-Teruel son prácticamente paralelas a las macroestructuras. Definen una clara alineación con la prolongación septentrional de la traza principal de la falla de Teruel (Peiro, 2016; Peiro et al., 2017). Las estriaciones encontradas en el afloramiento 1 (Fig. 2) indican una dirección de transporte hacia el ENE (estereogramas $1 a$, b en Fig. 2), coherente con los ejes de esfuerzos hipotéticos inferidos en el afloramiento 3 ( $\sigma_{1}$ : vertical; $\sigma_{3}: \mathrm{N} 050^{\circ} \mathrm{E}$; estereograma 3 en Fig. 2), con las trayectorias primarias de $\sigma_{3}$ del campo de esfuerzos más reciente (ENE-WSW; Simón, 1989; Arlegui et al., 2005), y también con las trayectorias del campo actual (Herraiz et al., 2000; De Vicente et al., 2008). Por ello, en este caso, no se puede discriminar claramente la influencia de cada uno de estos dos factores en el desarrollo de la fracturación.

No se han encontrado rupturas superficiales que quiebren transversalmente la zona de relevo entre las fallas de Concud y Teruel por el área de El Planizar (Fig. 2). Es por ello que se descarta la hipótesis de una incipiente conexión entre las fallas de Concud y Teruel por medio de una o varias fallas transversales de alto ángulo, características del modelo clásico de hard linkage (Walsh y Watterson, 1991). Por el contrario, Peiro (2016) y Peiro et al. (2017) determinan como más probable una hipotética futura unión a partir de la prolongación septentrional de la falla de Teruel hasta alcanzar la falla de Concud.

El análisis de la fracturación que afecta a la zona de relevo entre las fallas de Sierra Palomera y Concud arroja cierta luz sobre la influencia relativa de las macroestructuras y el campo de esfuerzos regional. La mayoría de las rupturas que afectan a los materiales más recientes (Neógeno y Cuaternario) están repartidas de manera homogénea por toda la zona de relevo y se disponen próximas a la perpendicular respecto a la dirección de extensión del campo de esfuerzos: N-S en los estereogramas $1 \mathrm{~b}, 3$ y en el diagrama en rosa 6 de la Fig. 4, y más cercanas a NNWSSE en los estereogramas $1 \mathrm{a}, 2$ y en el diagrama en rosa 7 de la Fig. 4. No hay ninguna evidencia superficial de fracturación transversal a la zona de relevo que implique hard linkage.

La evolución estructural de la zona de relevo entre las fallas de Calamocha y Sierra Palomera también parece estar poco influida por las macroestructuras. Las fallas que afectan a materiales cuaternarios conforman un sistema de grábenes de dirección predominante N-S (estereograma 5 en Fig. 5) que se expresan en el relieve en forma de lo que Gutiérrez et al. (1983) denominaron valles tectónicos. Las estrías presentes en estos planos de falla definen una dirección de transporte media hacia el WNW. La fracturación del afloramiento 1 (estereograma 1a y diagrama en rosa $1 \mathrm{~b}$ en Fig. 5) y de la falla de Bañón (estereogra- 

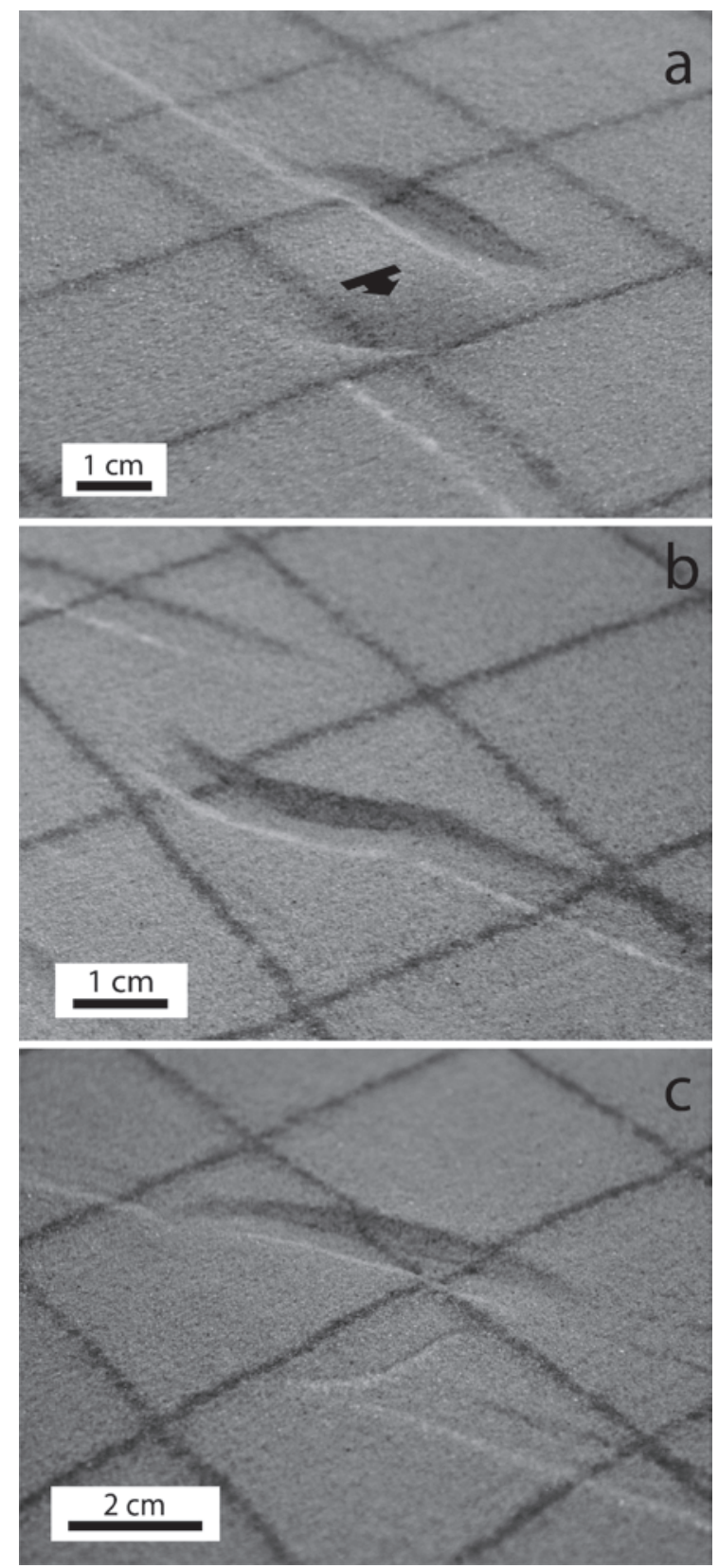

Figura 14. A) Relay ramp y falla transversa incipiente en B2-1. B) Prolongación "en gancho" de una estructura principal en B2-2. C) Falla transversa entre estructuras principales en B2-2.

Figure 14. A) Relay ramp and incipient transverse fault in B2-1. B) "Hooked" prolongation of a main structure in B2-2. C) Transverse fault between main structures in B2-2.

ma 3 en Fig. 5) viene determinada por las fallas a las que está asociada, ambas de dirección aproximada NNE-SSW. En cambio, la que afecta al Neógeno del afloramiento 2 (estereograma 2a y diagrama en rosa $2 \mathrm{~b}$ en Fig. 5) se caracteriza por una dirección predominante WSW-ENE. En este último caso, la extensión que ha dado lugar a las fracturas es ortogonal a la trayectoria primaria del campo de esfuerzos reciente, por lo que podría tratarse de un intercambio local entre los ejes $\sigma_{2}$ y $\sigma_{3}$, que es muy común en los regímenes de extensión radial o 'multidireccional' (Simón, 1989). En esta zona de relevo sí que existen estructuras claramente transversales que se localizan en su sector norte, y entre las que destaca la falla de Bañón (Fig. 5); pero éstas no parecen continuar hacia el sur, por lo que no se trataría de una unión completa por hard linkage.

\section{Respuesta de los modelos analógicos al régimen de extensión 'biaxial': aportaciones y evaluación del método}

La extensión 'biaxial' ha inducido la nucleación de fallas y grábenes asociados al borde de la silicona en las series 1 y 2 , además de un complejo sistema de grábenes centrales sobre todo en los modelos de la Serie 1.

Las estructuras principales asociadas al borde de la silicona han desarrollado zonas de relevo desde los estadios iniciales, favorecidas por la geometría escalonada en planta de la banda de silicona. Estas zonas de relevo se caracterizan por un suave basculamiento, que se va acentuando conforme avanzan los experimentos hasta los estadios intermedios (soft linkage; modelos B1-1, B2-1, B2-2, B2-3; Fig. 10A; Fig. 12A, B, C; Fig. 14A). Es en estos estadios intermedios cuando, en muchos casos, se han producido prolongaciones "en gancho" de las estructuras principales (modelos B1-2, B2-1, B2-1, B2-3; Fig. 10B; Fig. 12A, B, C; Fig. 14B). Algunas zonas de relevo se acaban fracturando transversalmente en estadios más avanzados, ya sea por planos de falla aislados (hard linkage; modelos B1-1, B2-1 y B2-2; Fig. 10A; Fig. 12A, B; Fig. $14 C)$ o por grábenes (modelo B2-3; Fig. 12C). Todas estas estructuras están más controladas por el campo de deformación que por la dirección de las fallas maestras que las limitan.

Aunque se desarrollen fuera de estas zonas de relevo, los sistemas de grábenes centrales, presentes sobre todo en los modelos de la Serie 1, también aportan información sobre el comportamiento de la fracturación bajo regímenes de esfuerzos inestables (Fig. 10). Son estructuras que se han desarrollado en puntos aislados desde estadios tempranos y poco a poco han ido propagándose y produciendo interacciones y uniones entre ellas. Su nucleación ha sido siempre desencadenada por la ascensión de diapiros de silicona, y presentan direcciones mayoritariamente controladas por el campo de deformación impuesto. 


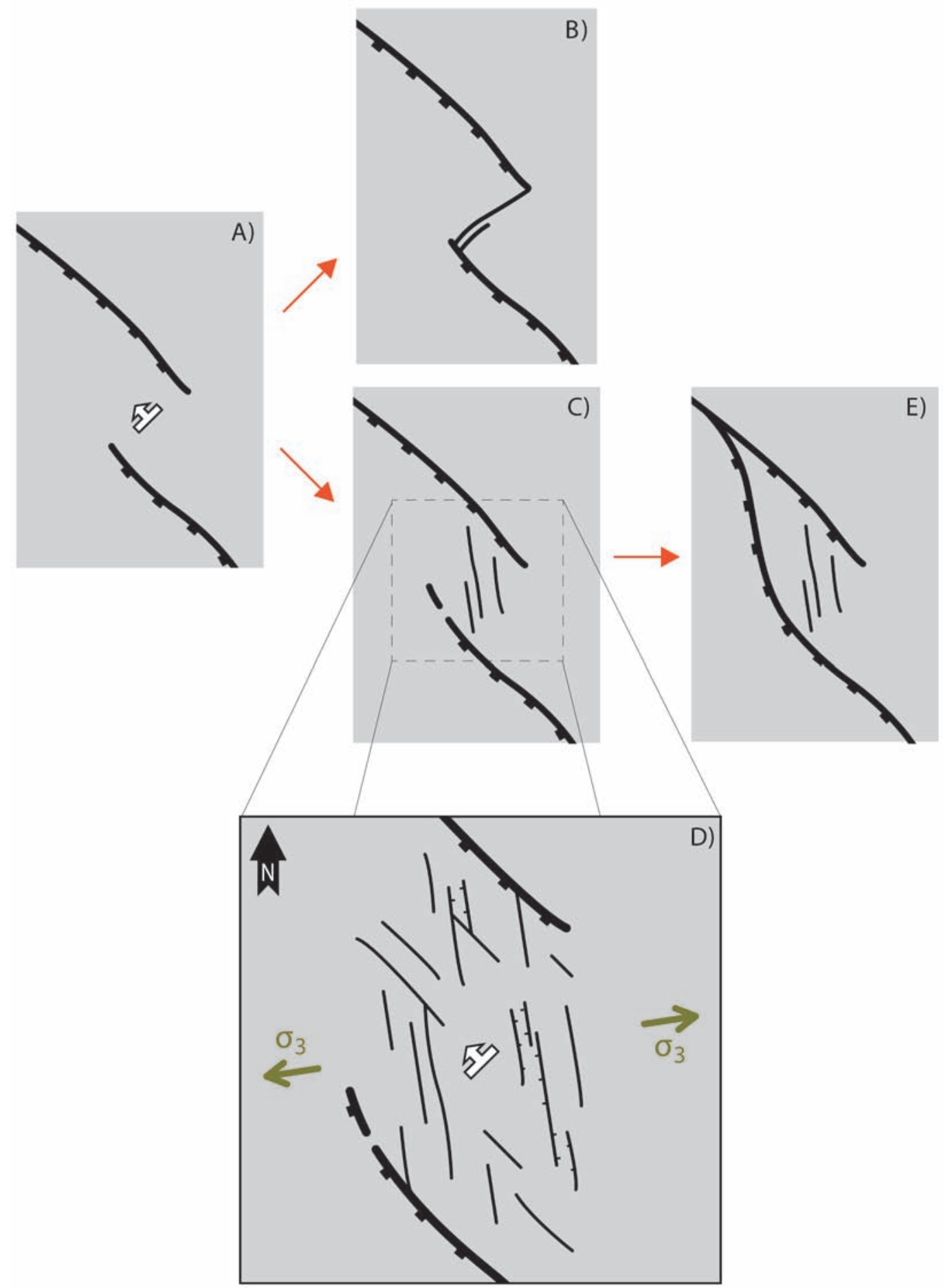

Figura 15. Tipos de interacción entre fallas a través de sus zonas de relevo: (A) y (B) soft y hard linkage, respectivamente (Walsh y Watterson, 1991); (C) relevo con fracturación longitudinal distribuida, que corresponde a las zonas de relevo entre las fallas del margen oriental de la fosa del Jiloca; (D) ampliación de la fracturación dispuesta preferentemente en dirección perpendicular al eje $\sigma_{3}$ del campo de esfuerzos reciente $y$, en menor medida, paralelas a las macroestructuras; (E) evolución futura hipotética de este último caso, que implica una unión de bajo ángulo alcanzada tras la propagación longitudinal de una de las fallas principales.

Figure 15. Types of interaction between faults through their relay zones: $(A)$ and $(B)$ soft and hard linkage, respectively (Walsh and Watterson, 1991); (C) relay with long-strike distributed fractures, which correspond to the relay zones between the faults of the eastern margin of the Jiloca basin; $(D)$ zoom to fractures with a mainly orthogonal orientation to the $\sigma_{3}$ axis of the recent stress field and, in a lesser extent, parallel to macrostructures; (E) hypothetical future evolution of the last context, which implies a low angle union by the longstrike prolongation of one main fault. 
Alba Peiro, et al., 2019. Zonas de relevo de falla en el margen oriental de la fosa del... Boletín Geológico y Minero, 130 (3): $393-415$

Los grábenes centrales son poco comunes en la Serie 2 (Fig. 12), donde predominan patrones de fracturación más controlados por las fallas principales. Ello sugiere que bajo un régimen de extensión más 'radial' las heterogeneidades locales inducidas por las fallas prefiguradas en la silicona tienen una importancia mayor, mientras que bajo una extensión más anisótropa o más 'uniaxial' (Serie 1; Fig. 8A, B) predomina una fracturación más homogénea controlada por el campo remoto. Aun con todo, hay que enfatizar que la fracturación que afecta tanto a las zonas de relevo generadas sobre los bordes de la silicona como a zonas cercanas a ellas está fuertemente controlada por el campo de deformación impuesto (Fig. 11 y 13).

Respecto al dispositivo experimental, éste ha permitido aplicar una extensión en dos direcciones perpendiculares y simular un campo de deformación heterogéneo, mediante la utilización de dos motores y una lámina de látex (Fig. 8). La geometría escalonada de la banda basal de silicona es la misma empleada por Hus et al. (2005), y ha permitido una correcta nucleación y localización de las fallas principales y, por lo tanto, de la deformación en sus zonas de relevo. Se han ensayado asimismo configuraciones como la de Le Calvez et al. (2002), que no han generado ningún tipo de deformación con este dispositivo.

\section{Comparación entre los modelos analógicos y la deformación del área de estudio}

De acuerdo con el dimensionamiento aplicado, la comparación entre los experimentos y la geología de la zona estudiada puede llevarse a cabo de manera fiable (Tabla 2). La relación de longitudes implica la formación de fallas principales de aproximadamente $10 \mathrm{~cm}$ de longitud en los modelos y $8 \mathrm{~km}$ en la naturaleza (o en el prototipo), así como un espaciado entre ellas de $2,5 \mathrm{~cm}$ en los modelos y $2 \mathrm{~km}$ en la naturaleza. Se trata de valores del mismo orden de magnitud a los de la zona de estudio. El escalado en profundidad implica un espesor de cobertera frágil de $1 \mathrm{~cm}$ en los modelos y $800 \mathrm{~m}$ en la naturaleza, así como un espesor del nivel de despegue de $0,5 \mathrm{~cm}$ en los modelos y $400 \mathrm{~m}$ en la naturaleza. Estos valores también son razonables considerando que las unidades dúctiles triásicas en la zona de estudio se encuentran muy deformadas y a profundidades que varían entre 0 y 800 m (Godoy et al., 1983; Olivé et al., 1983; Ramírez et al., 1983). Sin embargo, la extensión que ha sufrido el área de estudio es menor que la alcanzada en los experimentos (según se infiere de los desplazamientos totales producidos en las fallas exten- sionales de Teruel, Concud y Valdecebro; Simón et al., 2012). Es por ello que los estadios más avanzados de los experimentos pueden ser considerados como una posible predicción de la deformación futura en la zona de estudio.

Procesos de diapirismo como los asociados a los grábenes centrales de los modelos analógicos podrían ser también responsables de la distribución relativamente uniforme de los estrechos y numerosos grábenes paralelos que aparecen en la zona de relevo entre las fallas de Calamocha y Sierra Palomera (Fig. 5). Asimismo, en esta misma zona se han encontrado analogías con algunas de las estructuras asociadas a los grábenes centrales y con los procesos de interacción entre ellos, obtenidas a partir de la modelización analógica. Destaca sobre todo la formación de rampas de relevo a diferentes escalas.

En los modelos analógicos se ha visto cómo las zonas de relevo entre fallas se mantienen prácticamente intactas hasta los estadios más avanzados, en los que solo algunas acaban fracturándose transversalmente. En el área de estudio no se han encontrado evidencias de rupturas transversales que impliquen una unión completa de las macroestructuras por una o varias fallas transversales (hard linkage). Sin embargo, son zonas con abundante desarrollo de fracturación dispuesta en dos direcciones: paralelas a las fallas principales $y$, sobre todo, perpendiculares a las trayectorias primarias del eje $\sigma_{3}$ correspondientes al campo de esfuerzos más reciente.

A partir de los resultados de los modelos analógicos y del análisis estructural puede valorarse que la situación de las zonas de relevo entre las fallas de Teruel, Concud, Sierra Palomera y Calamocha podría representar un estadio intermedio entre la independencia completa y la coalescencia de las fallas involucradas, como ya se infirió entre las de Concud y Teruel (Lafuente et al., 2011; Peiro, 2016; Peiro et al., 2017). Se trata, a su vez, de un tipo de interacción entre fallas diferente del soft linkage (Fig. 15A) y del hard linkage (Fig. 15B), denominada en este trabajo interacción por relevo con fracturación longitudinal distribuida (Fig. 15C). Ésta se caracteriza por un tipo de fracturación esencialmente longitudinal a las fallas maestras, controlada en parte por la dirección de éstas $y$, sobre todo, por el campo de esfuerzos remoto o primario, con muy poca presencia de fracturación transversal (Fig. 15D). La evolución futura de este tipo de zona de relevo no es previsible que siga el modelo clásico de hard linkage (con falla transversal), sino una unión por propagación longitudinal de una de las fallas hasta unirse a la otra (Fig. 15E). 
Alba Peiro, et al., 2019. Zonas de relevo de falla en el margen oriental de la fosa del... Boletín Geológico y Minero, 130 (3): $393-415$

\section{Conclusiones}

La fosa del Jiloca es una depresión alargada cuyo margen oriental está formado por tres grandes fallas normales de dirección NW-SE con una disposición escalonada diestra: fallas de Calamocha, Sierra Palomera y Concud. Junto con la falla deTeruel, constituyen un conjunto de estructuras activas desde el Plioceno hasta la actualidad, y determinan tres zonas de relevo con evidencias de fracturación reciente. Estas fallas se mueven bajo un campo extensional radial o 'multidireccional' con trayectorias primarias del eje $\sigma_{3}$ ENE-WSW.

Cada zona de relevo de falla acomoda la transferencia de desplazamiento mediante un basculamiento o relay ramp, lo que constituye una primera evidencia de interacción entre ellas por soft linkage. A ello se añade, en las tres zonas, una fracturación que se reparte de manera bastante homogénea. El análisis de fracturas que afectan a los materiales más recientes (Mioceno Superior- Cuaternario) ha permitido identificar dos direcciones preferentes: una longitudinal a las fallas principales que delimitan los relevos (NW-SE) y otra, más frecuente, coherente con el campo de esfuerzos (cercana a N-S). La presencia de fracturación transversal a las zonas de relevo es prácticamente nula, por lo que no existen evidencias de hard linkage.

El estudio de los modelos analógicos ha permitido identificar las variables que influyen en el desarrollo de la fracturación en las zonas de relevo. Se han llevado a cabo dos series de modelos bajo dos campos de esfuerzos diferentes, mediante variaciones en las velocidades de dos motores dispuestos ortogonalmente. Los modelos sometidos al régimen menos 'radial' (Serie 1) muestran un desarrollo abundante de deformación en las fallas principales y también en zonas cercanas a ellas (grábenes centrales). La mayoría de estas rupturas están controladas por el campo de deformación impuesto, siendo la influencia de la orientación de las fallas principales prácticamente nula. Los modelos sometidos al régimen más 'radial' (Serie 2) concentran la deformación en las fallas principales y en sus zonas de relevo, desarrollando estructuras transversales sólo en los estadios más avanzados de los experimentos. Asimismo, dentro de cada serie se ha corroborado que la influencia del campo de deformación es siempre mayor que la de las fallas maestras.

Las fallas mayores interaccionan entre sí mediante la fracturación longitudinal que se desarrolla en sus zonas de relevo, que está controlada por la dirección de las mismas y, en mayor medida, por el campo de extensión radial activo en este sector de la
Cordillera Ibérica. Denominada en este trabajo interacción por relevo con fracturación longitudinal distribuida, constituye un tipo diferente del soft linkage y del hard linkage definidos en la literatura. Su evolución futura no es previsible que dé lugar a una unión de tipo hard linkage, sino a una conexión de bajo ángulo alcanzada tras la propagación longitudinal (paralela o ligeramente oblicua) de una de las fallas.

\section{Referencias}

Acocella, V., Morvillo, P., Funiciello y R. 2005. What controls relay ramps and transfer faults within rift zones? Insights from analogue models. Journal of Structural Geology, 27, 397-408.

Álvaro, M., Capote, R. y Vegas, R. 1979. Un modelo de evolución geotectónica para la Cadena Celtibérica. Acta Geológica Hispánica, 14, 172-177.

Arlegui, L.E., Simón, J.L., Lisle, R.J. y Orife, T. 2005. Late Pliocene-Pleistocene stress field in the Teruel and Jiloca grabens (eastern Spain): contribution of a new method of stress inversion. Journal of Structural Geology, 28, 1019-1027.

Anderson, E.M. 1951. The dynamics of faulting and dyke formation with application to Britain. Oliver \& Boyd, Edimburgh, $206 \mathrm{p}$.

Bahroudi, A., Hemin, A.K. y Talbot, C.J. 2003. Effect of ductile and frictional dècollements on style of extension. Journal of Structural Geology, 25, 1401-1423.

Capote, R., Gutiérrez, M., Hernández, A. y Olivé A. 1981. Movimientos recientes de la fosa del Jiloca (Cordillera Ibérica). V Reunión del Grupo Español de Trabajo del Cuaternario, Sevilla, 245-257.

Clifton, A.E., Schlische, R.W., Withjack, M.O. y Ackermann, R.V. 2000. Influence of rift obliquity on fault-population systematics: results of experimental clay models. Journal of Structural Geology, 22, 1491-1509.

De Vicente, G., Cloetinghm S., Muñoz-Martín, A., Olaiz, A., Stich, D., Vegas, R., Galindo-Zaldívar, J. y FernándezLozano, J. 2008. Inversion of moment tensor focal mechanisms for active stresses around the microcontinent Iberia: Tectonic implications. Tectonics, 27, DOI: 10.1029/2006TC002093

Ezquerro, L. 2017. El sector norte de la cuenca neógena de Teruel: tectónica, clima y sedimentación. Tesis Doctoral, Universidad de Zaragoza, 494 p.

García-Lacosta, A.l., Pueyo, Ó., Arlegui, L.E., Liesa, C.L., Ezquerro, L. y Simón, J.L. 2014. La zona de falla reciente de Sierra Palomera (fosa del Jiloca, Cordillera Ibérica): contribución de la geofísica a la caracterización estructural. 2a Reunión Ibérica sobre Fallas Activas y Paleosismología, Lorca, 51-54.

Godoy, A., Ramírez, J.I., Olivé, A., Moissenet, E., Aznar, J.M., Aragonés, E., Aguilar, M.J., Ramírez del Pozo, J., Leal, M.C., Jerez Mir, L., Adrover, R., Goy, A., Comas, M.J., Alberdi, M.T., Giner, J., Gutiérrez Elorza, M., Portero, 
Alba Peiro, et al., 2019. Zonas de relevo de falla en el margen oriental de la fosa del... Boletín Geológico y Minero, 130 (3): $393-415$

J.M. y Gabaldón, V. 1983. Mapa Geológico de España 1: 50.000, hoja $n^{\circ} 567$ (Teruel). IGME, Madrid.

Gutiérrez, M., Peña, J.L. y Simón, J.L. 1983. Los valles tectónicos recientes de Rubielos de la Cérida (Teruel). Actas VI Reunión Nacional Grupo Español de Trabajo del Cuaternario, Vigo-Santiago de Compostela, 449-459.

Gutiérrez, F., Gutiérrez, M., Gracia, F.J., McCalpin, J.P., Lucha, P. y Guerrero J. 2008. Plio-Quaternary extensional seismotectonics and drainage network development in the central sector of the Iberian Chain (NE Spain). Geomorphology, 102, 21-42.

Hernández, A., Olivé, A., Moissenet, E., Pardo, G., Villena, J., Portero, J.M., Gutiérrez, M., Puigdefábregas, C., Giner, J., Aguilar, M.J., Leal, M.C., Gutiérrez, J.C., Gil, M.D., Adrover, R. y Gabaldón, V. 1983. Mapa Geológico de España 1: 50.000, hoja $n^{\circ} 491$ (Calamocha). IGME, Madrid.

Herraiz, M., De Vicente, G., Lindo-Ñaupari, R., Giner, J., Simón, J.L., González-Casado, J.M., Vadillo, O., Rodríguez-Pascua, M.A., Cicuéndez, J.I., Casas, A., Cabañas, L., Rincón, P., Cortés, A.L., Ramírez, M., Lucini, M. 2000. The recent (upper Miocene to Quaternary) and present tectonic stress distributions in the Iberian Peninsula. Tectonics, 19, 762-786.

Hubbert, M. K. 1937. Theory of scale models as applied to the study of geologic structures. Bulletin of the Geological Society of America, 48, 1459-1519.

Hus, R., Acocella, V., Funiciello, R. y De Batist, M. 2005. Sandbox models of relay ramp structure and evolution. Journal of Structural Geology, 27, 459-473.

Koyi, H. 1988. Experimental modelling of role of gravity and lateral shortening in Zagros mountain Belt. The American Association of Petroleum Geologists Bulletin, 72, 1381-1394.

Krantz, R.W. 1991. Measurements of friction coefficients and cohesion for faulting and fault reactivation in laboratory models using sand and sand mixtures. Tectonophysics, 188, 203-207.

Lafuente, P., Arlegui, L.E., Casado, I., Ezquerro, L., Liesa, C.L., Pueyo, Ó. y Simón, J.L. 2011. Geometría y cinemática de la zona de relevo entre las fallas neógeno-cuaternarias de Concud y Teruel (Cordillera Ibérica). Revista de la Sociedad Geológica de España, 24, 109-125.

Le Calvez, J. H., Bruno, C. y Vendeville, C. 2002. Experimental designs to model along-strike fault interaction. Journal of the Virtual Explorer, 7, 1-17.

Liesa, C.L., Simón, J.L., Ezquerro, L., Arlegui, L.E. y Luzón, A. 2019. Stress evolution and structural inheritance controlling an intracontinental extensional basin: The central-northern sector of the Neogene Teruel Basin. Journal of Structural Geology, 118, 362-376.

Mandl, G., De-Jong, L. N. J. y Maltha, A. 1977. Shear zones in granular material; an experimental study of their structure and mechanical genesis. Rock Mechanics, 9, 95-144.

Mansfield, C. y Cartwright, J. 2001. Fault growth by linkage: observations and implications from analogue models. Journal of Structural Geology, 23, 745-763.
Martín-Bello, L., Arlegui, L.E., Ezquerro, L., Liesa, C.L. y Simón, J.L. 2014. La falla de Calamocha (fosa del Jiloca, Cordillera Ibérica): estructura y actividad pleistocena. $2^{a}$ Reunión Ibérica sobre Fallas Activas y Paleosismología, Lorca, 55-58.

Olivé, A., Hernández, A., Moissenet, E., Pardo, G., Villena, J., Gutiérrez, M., Puigdefábregas, C., Giner, J., Aguilar, M.J., Leal, M.C., Goy, A., Comas, M.J., Adrover, R., Portero, J.M. y Gabaldón, V. 1983. Mapa Geológico de España 1: 50.000, hoja $n^{\circ} 516$ (Monreal del Campo). IGME, Madrid.

Peiro, A. 2016. Una posible prolongación septentrional de la falla de Teruel y su interacción con la falla de Concud. Trabajo Fin de Grado, Universidad de Zaragoza, 36 p.

Peiro, A., Simón, J.L. y Liesa, C.L. 2017. New evidence of recent fracturing at the relay zone between the Concud and Teruel faults (eastern Iberian Chain). Geogaceta, 62, $115-118$.

Ramberg, H. 1981. Gravity, Deformation and the Earth's Crust in Theory, Experiments and Geological Applications. Academic Press, London, 452 p.

Ramírez, J.I., Olivé, A., Moissenet, E., Aragonés, E., Ramírez, J., Leal, M.C., Aguilar, M.J., Adrover, R., Giner, J., Gutiérrez, J.C., Goy, A., Comas, M.J., Portero, J.M. y Gabaldón, V. 1983. Mapa Geológico de España 1: 50.000, hoja 541 (Santa Eulalia). IGME, Madrid.

Rubio, J.C. y Simón, J.L. 2007. Tectonic subsidence vs. Erosional lowering in a controversial intramontane depression: the Jiloca basin (Iberian Chain, Spain). Geological Magazine, 144, 1-15.

Schellart, W.P. 2000. Shear test results for cohesion and friction coefficients for different granular materials: scaling implications for their usage in analogue modelling. Tectonophysics 324, 1-16.

Sharma, P.V. 1997. Environmental and Engineering Geophysics. Cambridge University Press, Cambridge, 475 p. DOI:10.1017/CB09781139171168.

Simón, J.L. 1982. Compresión y distensión alpinas en la Cadena Ibérica Oriental. Tesis Doctoral, Universidad de Zaragoza, 269 p.

Simón, J.L. 1983. Tectónica y neotectónica del sistema de fosas de Teruel. Teruel, 69, 21-97.

Simón, J.L. 1989. Late Cenozoic stress field and fracturing in the Iberian Chain and Ebro Basin (Spain). Journal of Structural Geology, 11, 285-294.

Simón, J.L., Arlegui, L., Lafuente, P. y Liesa, C.L. 2012. Active extensional faults in the central-eastern Iberian Chain, Spain. Journal of Iberian Geology, 38. 127-144.

Simón, J.L., Arlegui, L.E., Ezquerro, L., Lafuente, P., Liesa, C.L. y Luzón, A. 2016. Enhaced paleoseismic succession at the Concud Fault (Iberian Chain, Spain): new insights for seismic hazard assessment. Natural Hazards, 80, 1967-1993.

Simón, J.L., Arlegui, L.E., Ezquerro, L., Lafuente, P., Liesa, C.L. y Luzón, A. 2017. Structure and paleoseismology of the Teruel Fault: dynamic interaction and strain partitioning with the Concud Fault (eastern Iberian Chain, Spain). Journal of Structural Geology, 103, 100-119.

Vendeville, B., Cobbold, P.R., Davy, P., Brun, J.P., y 
Choukroune, P. 1987. Physical models of extensional tectonics at various scales. En: Continental Extensional Tectonics (Coward, M.P., Dewey, M.P., y Hancock, P.L., Eds), Geological Society of London, Spec. Publ. 28, 95107.

Walsh, J.J. y Watterson, J. 1991. Geometric and kinematic coherence and scale effects in normal fault systems.
Geological Society of London, Special Publications, 56, 193-203.

Weijermars, R. y Schmeling, H. 1986. Scaling of Newtonian and non-Newtonian fluid dynamics without inertia for quantitative modelling of rock flow due to gravity (including the concept of rheological similarity). Physics of the Earth Planetary Interior, 43, 316-330.

Recibido: septiembre 2018

Revisado: enero 2019

Aceptado: marzo 2019

Publicado: septiembre 2019 
